\title{
Cohomogeneity one disk bundles with normal homogeneous collars
}

\author{
Lorenz J. Schwachhöfer*, Kristopher Tapp
}

\begin{abstract}
We consider cohomogeneity one homogeneous disk bundles and adress the question when these admit a nonnegatively curved 1 invariant metric with normal collar, i.e., such that near the boundary the metric is the product of an interval and a normal homogeneous space. If such a bundle is not (the quotient of) a trivial bundle, then we show that its rank has to be in $\{2,3,4,6,8\}$. Moreover, we give a complete classification of such bundles of rank 6 and 8 , and a partial classification for rank 3 .
\end{abstract}

\section{Introduction}

The search for manifolds of nonnegative curvature is one of the classical problems in Riemannian geometry. One source of examples has been compact Lie groups and their quotients, including homogeneous spaces and biquotients. In addition to these, one has examples formed by glueing two manifolds along a common boundary, first done by J. Cheeger ([C]). For a detailed survey on the known techniques and examples, we refer to [Z].

A large family of nonnegatively curved metrics was recently obtained by K. Grove and W. Ziller, who investigated closed cohomogeneity one manifolds with two singular orbits ([GZ]). Each such manifold is obtained by the glueing along a principal orbit of two cohomogeneity one homogeneous vector bundles, i.e., bundles of the form

$$
M:=G \times_{K} V \longrightarrow G / K,
$$

where $K \subset G$ are compact Lie groups, and $K$ acts transitively on the unit sphere of a finite dimensional Euclidean vector space $V$ by some orthogonal representation, with isotropy group henceforth denoted $H \subset K$. Grove and Ziller showed that any cohomogeneity one homogeneous vector bundle of rank at most two (that is, $\operatorname{dim}_{\mathbb{R}} V \leq 2$ ) admits an invariant nonnegatively-curved metric with a normal homogeneous collar, i.e., outside a compact set, the metric is $G$-equivariantly isometric to the Riemannian product of an interval and $G / H$

${ }^{*}$ Supported by the Schwerpunktprogramm Differentialgeometrie of the Deutsche Forschungsgesellschaft

${ }^{1}$ Throughout this article, the term "curvature" refers to the sectional curvature. 
with a normal homogeneous metric. Thus, any cohomogeneity one manifold whose singular orbits are of codimension at most two admits a $G$-invariant metric of nonnegative curvature.

In this article, we address the question of which cohomogeneity one homogeneous vector bundles of rank higher than two admit invariant metrics with nonnegative curvature and normal homogeneous collar. This question is of interest because, for a compact cohomogeneity one manifold $N$ with group diagram $H \subset\left\{K_{+}, K_{-}\right\} \subset G$, if the vector bundles associated to both halves of this diagram admit such metrics, then $N$ admits an invariant nonnegatively curved metric with normal homogeneous principle orbits in the middle. Not all bundles admit such metrics because there are cohomogeneity one manifolds which are known not to admit any invariant nonnegatively-curved metric (GVWZ]).

One class of bundles which does admit nonnegatively curved $G$-invariant metrics with normal homogeneous collar are what we call essentially trivial bundles by which we mean bundles of the form

$$
M:=(G \times L) \times_{K \times L} V \longrightarrow G / K,
$$

where the action of $\{1\} \times L$ on $V$ is transitive on the unit sphere. Thus, essentially trivial bundles are quotients of the trivial bundle $G \times V=(G \times L) \times{ }_{L} V$ under the action of a subgroup $K \subset G$, so that the existence of such a metric follows from a constuction in [STu]; cf. Corollary 2.2 for details. For example, all cohomogeneity one homogeneous vector bundles of rank one are essentially trivial.

Apart from these, our results show that bundles admitting such metrics are scarce.

Theorem 1.1 Let $M:=G \times_{K} V \rightarrow G / K$ be a cohomogeneity one homogeneous vector bundle which is essentially non-trivial. If $M$ admits a G-invariant metric with nonnegative curvature and normal homogeneous collar, then the rank of this bundle must be in $\{2,3,4,6,8\}$.

As was previously mentioned, all rank two bundles admit a $G$-invariant metric of nonnegative curvature and normal homogeneous collar by [GZ]. In the higher rank case, the situation is much more restricted. For rank eight, we have the following complete classification.

Theorem 1.2 Let $M:=G \times_{K} V \rightarrow G / K$ be a $G$-irreducible cohomogeneity one homogeneous vector bundle which is essentially non-trivial and such that $\operatorname{dim}_{\mathbb{R}} V=8$. Then $M$ admits a G-invariant metric of nonnegative curvature with normal homogeneous collar if and only if $M$ is finitely $G$-equivariantly covered by one of the following:

1. $\operatorname{Spin}(p+9) \times_{\operatorname{Spin}(8)} \mathbb{R}^{8}$ for $p \in\{0,1,2\}$, where $\operatorname{Spin}(8)$ acts on $\mathbb{R}^{8}$ by a spin representation, and $\operatorname{Spin}(8) \subset \operatorname{Spin}(p+9)$ is the lift of the standard inclusion $S O(8) \subset S O(p+9)$.

2. $\operatorname{Spin}(p+8) \times_{\operatorname{Spin}(7)} \mathbb{R}^{8}$ for $p \in\{0,1\}$, where $\operatorname{Spin}(7)$ acts on $\mathbb{R}^{8}$ by the spin representation, and $\operatorname{Spin}(7) \subset \operatorname{Spin}(p+8)$ is the lift of the standard inclusion $S O(7) \subset S O(p+8)$.

3. $\operatorname{Spin}(7) \times_{\operatorname{Spin}(6)} \mathbb{C}^{4}$, with the standard representation of $\operatorname{Spin}(6) \cong S U(4)$ on $\mathbb{C}^{4}$. 
4. A quotient of one of the preceding examples:

(a) $\left(\operatorname{Spin}(p+9) \cdot G^{\prime}\right) \times_{\operatorname{Spin}(8) \cdot H^{\prime}} \mathbb{R}^{8}$ for $p \in\{1,2\}$ and an arbitrary compact Lie group $G^{\prime}$ and $H^{\prime} \subset \operatorname{Spin}(p+1) \cdot G^{\prime}$ with $H^{\prime} \not \subset G^{\prime}$, which acts trivially on $\mathbb{R}^{8}$.

(b) $\left(\operatorname{Spin}(9) \cdot G^{\prime}\right) \times_{\operatorname{Spin}(7) \cdot H^{\prime}} \mathbb{R}^{8}$ for an arbitrary compact Lie group $G^{\prime}$ and $H^{\prime} \subset$ $\operatorname{Spin}(2) \cdot G^{\prime}=S^{1} \cdot G^{\prime}$ with $H^{\prime} \not \subset G^{\prime}$, which acts trivially on $\mathbb{R}^{8}$.

(c) $\left(\operatorname{Spin}(7) \cdot G^{\prime}\right) \times_{\operatorname{Spin}(6) \cdot S^{1} \cdot H^{\prime}} \mathbb{C}^{4}$ for an arbitrary compact Lie group $G^{\prime} \supset S^{1} \cdot H^{\prime}$, where $S^{1} \subset G^{\prime}$ acts on $\mathbb{C}^{4}$ by multiples of the identity, and $H^{\prime} \subset G^{\prime}$ acts trivially.

Here, for Lie groups $L_{1}, L_{2}$, we denote by $L_{1} \cdot L_{2}$ the quotient of $L_{1} \times L_{2}$ by a finite subgroup of the center. The term $G$-irreducible means that $M$ is not $G$-equivariantly finitely covered by a bundle of the form $\left(G_{1} / H_{1}\right) \times M^{\prime}$ with $\operatorname{dim}\left(G_{1} / H_{1}\right)>0$ and $M^{\prime}$ a cohomogeneity one homogeneous vector bundle. This hypothesis is natural because, for $G$-reducible bundles, our problem easily reduces to deciding whether $M^{\prime}$ admits such a metric.

Glueing together two disk bundles of the second type of Theorem 1.2 with $p=0$, after applying outer automorphisms of $\operatorname{Spin}(8)$, we conclude that the primitive cohomogeneity one manifold given by the group diagram $G_{2} \subset\left\{\operatorname{Spin}_{+}(7), \operatorname{Spin}_{-}(7)\right\} \subset \operatorname{Spin}(8)$ admits a metric of nonnegative curvature with a totally geodesic normal homogeneous principal orbit. However, this manifold is diffeomorphic to the sphere $S^{15}$ ([GWZ]).

For rank 6 bundles, we obtain the following

Theorem 1.3 Let $M:=G \times_{K} V \rightarrow G / K$ be a $G$-irreducible cohomogeneity one homogeneous vector bundle which is essentially non-trivial and such that $\operatorname{dim}_{\mathbb{R}} V=6$. Then $M$ admits a G-invariant metric of nonnegative curvature with normal homogeneous collar if and only if $M$ is finitely $G$-equivariantly covered by one of the following:

1. $S U(5) \times_{S U(4)} \mathbb{R}^{6}$, with the irreducible action of $S U(4) \cong \operatorname{Spin}(6)$ on $\mathbb{R}^{6}$.

2. $\left(S U(5) \cdot G^{\prime}\right) \times_{S U(4) \cdot H^{\prime}} \mathbb{R}^{6}$ for an arbitrary compact Lie group $G^{\prime}$ and $H^{\prime} \subset S^{1} \cdot G^{\prime}$ with $S^{1} \subset S U(5)$ being the centralizer of $S U(4)$, and $H^{\prime} \not \subset G^{\prime}$ acts trivially on $\mathbb{R}^{6}$.

To describe our results for rank three and four bundles, we require some notation for subgroups of the exceptional Lie group $G_{2}$. Let $S O(4) \subset G_{2}$ and $S U(3) \subset G_{2}$ be the isotropy groups of the symmetric space $G_{2} / S O(4)$ and the sphere $S^{6}=G_{2} / S U(3)$, respectively. After conjugating these groups appropriately, their intersection can be made isomorphic to $U(2)$, and we let $S U(2)_{1} \subset S O(4) \cap S U(3) \subset G_{2}$ be the simple part of this intersection. Note that $S U(2)_{1} \subset S O(4)$ is normal, and we denote its centralizer in $G_{2}$ by $S U(2)_{3} \subset S O(4) \subset G_{2}$. (The subscripts of the $S U(2)$-subgrous of $S O(4)$ denote their maximal weight for the isotropy representation of $G_{2} / S O(4)$.) Using this notation, we can make the following statement about the rank three case.

Theorem 1.4 Let $M:=G \times{ }_{K} V$ be a $G$-irreducible cohomogeneity one homogeneous vector bundle which is essentially non-trivial such that $\operatorname{dim}_{\mathbb{R}} V=3$. If $M$ admits a nonnegativelycurved $G$-invariant metric with normal homogeneous collar, then $M$ must be finitely $G$ equivariantly covered by one of the following. 
1. $M_{1}=G_{2} \times_{S O(4)} \mathfrak{s u}(2)_{3}$, where $S O(4)$ acts on $\mathfrak{s u}(2)_{3} \triangleleft \mathfrak{s o}(4)$ by the adjoint representation.

2. $M_{2}=\left(S p(p+1) \cdot G^{\prime}\right) \times_{S p(1) \cdot H^{\prime}} \mathfrak{s p}(1)$ with $H^{\prime} \subset S p(p) \cdot G^{\prime}$, where $S p(1) \cdot H^{\prime}$ acts on $\mathfrak{s p}(1) \triangleleft \mathfrak{s p}(1) \oplus \mathfrak{h}^{\prime}$ by the adjoint representation.

Further, $M_{1}$ admits such a metric, as does $M_{2}$ with $G^{\prime}=1$ and $H^{\prime}=S p(p)$.

Finally, in the rank four case, we have the following examples, which are all related to those in Theorem 1.4.

Theorem 1.5 The following cohomogeneity one homogeneous vector bundles (orbifold bundles, respectively) of rank four admit $G$-invariant metrics of nonnegative curvature and normal homogeneous collar:

1. $G_{2} \times_{S O(4)}(\mathbb{H} / \pm 1)$, where $S U(2)_{1} \subset S O(4)$ acts trivially and $S U(2)_{3} \subset S O(4)$ by left multiplication on $\mathbb{H} / \pm 1$. Note that this is an orbifold bundle only.

2. $\left(G_{2} \times G^{\prime}\right) \times_{S O(4) \times S U(2)^{\prime}}(\mathbb{H} / \pm 1)$, where $S U(2)_{1} \subset S O(4)$ acts trivially and $S U(2)_{3} \subset$ $S O(4)$ by left multiplication, whereas $S U(2)^{\prime} \subset G^{\prime}$ acts by right multiplication on $\mathbb{H} / \pm 1$ with $G^{\prime}$ arbitrary. Note that these are orbifold bundles only.

3. $S p(p+1) \times_{S p(1) \times S p(p)} \mathbb{H}$ where $S p(p)$ acts trivially and $S p(1)$ by left multiplication on $\mathbb{H}$.

4. $\left(S p(p+1) \times G^{\prime}\right) \times_{S p(1) \times S p(p) \times S p(1)^{\prime}} \mathbb{H}$ where $S p(p)$ acts trivially and $S p(1)$ by left multiplication, whereas $S p(1)^{\prime} \subset G^{\prime}$ acts by right multiplication on $\mathbb{H}$ with $G^{\prime}$ arbitrary.

It is a pleasure to thank Karsten Grove and Wolfgang Ziller for many inspiring and clarifying discussions on this work. We are also grateful to the American Institute of Mathematics for hospitality and funding at a workshop on nonnegative curvature in September 2007, where portions of this work were discussed.

\section{Construction of metrics}

In this section, we construct invariant nonnegatively curved metrics with normal homogeneous collars on all of the bundles which are claimed to admit such metrics in Theorems $1.2,1.3] 1.4,1.5$,

We adopt the following notation for the remainder of the article. Let $M:=G \times_{K} V \rightarrow$ $G / K$ be a cohomogeneity one homogeneous vector bundle, and let $H \subset K$ denote the isotropy group of the transitive action of $K$ on the unit sphere in $V$. Let $\mathfrak{h} \subset \mathfrak{k} \subset \mathfrak{g}$ denote the Lie algebras of $H \subset K \subset G$, and let $Q$ be an Ad-invariant inner product on $\mathfrak{g}$. We denote the $Q$-orthogonal decompositions by

$$
\mathfrak{k}=\mathfrak{h} \oplus \mathfrak{m}, \quad \mathfrak{g}=\mathfrak{k} \oplus \mathfrak{s}, \quad \text { and we let } \mathfrak{p}:=\mathfrak{m} \oplus \mathfrak{s} .
$$

All of our examples come from: 
Theorem 2.1 If there exists $C>0$ such that for all $X=X_{\mathfrak{m}}+X_{\mathfrak{s}}, Y=Y_{\mathfrak{m}}+Y_{\mathfrak{s}} \in \mathfrak{p}$ we have the inequality

$$
\left|X_{\mathfrak{m}} \wedge Y_{\mathfrak{m}}\right| \leq C|[X, Y]|
$$

then $M$ admits a nonnegatively curved $G$-invariant metric with normal homogeneous collar.

Proof. In [STu, Theorem 5.1], it was shown that for each linear action of $K$ on $V$ which is transitive on the unit sphere $S^{l} \subset V$, there exists a nonnegatively curved $K$-invariant metric on $V$ which outside a compact set has the form $g_{V}=d t^{2}+f(t)^{2} g_{Q}^{S^{l}}$ where $g_{Q}^{S^{l}}$ is the normal homogeneous metric on $S^{l}$ induced by the bi-invariant metric $Q$ on $K$, and $f$ is a smooth function with $f^{\prime}>0$. From the curvature formula for warped products it follows that $f^{\prime \prime} \leq 0$, and that this metric remains nonnegatively-curved if we replace $f$ by any other function with $f^{\prime \prime} \leq 0$. Therefore, we can achieve that $f$ is constant for large $t$. Thus, we may assume that $g_{V}$ has the form

$$
g_{V}=d t^{2}+c_{0}^{2} g_{Q}^{S^{l}}
$$

outside $B_{R}(0) \subset V$ for some $R>0$, where $c_{0}>0$ can be chosen to be arbitrarily large.

Next, let $g_{\varepsilon}$ be the left-invariant metric on $G$ given by

$$
g_{\varepsilon}=\left.Q\right|_{\mathfrak{s}}+\left.(1+\varepsilon) Q\right|_{\mathfrak{k}} .
$$

Then $K$ acts by isometries on $\left(G \times V, g_{\varepsilon}+g_{V}\right)$ as $k \star(g, v):=\left(g k^{-1}, k v\right)$. There is a unique induced metric on $M$ such that the canonical submersion $G \times V \rightarrow M$ is Riemannian. This metric on $M$ is invariant under the $G$-action induced from the left action on the first factor of $G \times V$.

Furthermore, it was shown in [STa, Theorem 0.2] that under our hypothesis, the tangent planes in $\mathfrak{p}=\mathfrak{s} \oplus \mathfrak{m} \subset \mathfrak{g}$ have nonnegative curvature for $\left(G, g_{\varepsilon}\right)$ for sufficiently small $\varepsilon>0$. Thus, the horizontal planes of the submersion $\left(G \times V, g_{\varepsilon}+g_{V}\right) \rightarrow M$ all have nonnegative curvature, hence so does the induced metric on $M$ by O'Neill's formula.

If we choose $c_{0}^{2}:=(1+\varepsilon) / \varepsilon$, then it follows from the "scale-up/scale-down" metric construction of [GZ, Lemma 2.1] that $M$ has a normal homogeneous collar.

Corollary 2.2 Let $M=G \times{ }_{K} V \rightarrow G / K$ be a cohomogeneity one homogeneous disc bundle for which (2) holds, and let $G^{\prime}$ be an arbitrary compact Lie group. Let $K^{\prime} \subset \operatorname{Norm}_{G}(K) \times G^{\prime}$ be a closed subgroup containing $K \cong K \times\{1\}$, and let $K^{\prime}$ act orthogonally on $V$ extending the action of $K \subset K^{\prime}$. Then the bundle

$$
M^{\prime}:=\left(G \times G^{\prime}\right) \times_{K^{\prime}} V=\left(M \times G^{\prime}\right) /\left(K^{\prime} / K\right)
$$

admits an invariant metric of nonnegative curvature and normal homogeneous collar. In particular, this shows that every essentially trivial homogeneous disk bundles admits such a metric. 
Proof. We assert that the $K$-invariant metric on $V$ from [STu, Theorem 5.1] used in the proof of Theorem 2.1 is invariant not only under $K$ but under $\operatorname{Norm}_{O(V)} K$. This is due to the fact that for all but one type of transitive actions on spheres ([MS]; cf Table 1), the isotropy representation splits into mutually inequivalent irreducible representations, so that any $K$-invariant metric on $V$ is $\operatorname{Norm}_{O(V)}(K)$-invariant by Schur's Lemma. The only exception is $S^{4 p+3}=S p(p+1) / S p(p)$ in which case the isotropy has a 3-dimensional trivial summand, and $\operatorname{Norm}_{O(4 p+4)} S p(p)=S p(1) \cdot S p(p)$. However, looking at the construction in [STu], it follows that this metric is $\operatorname{Norm}_{O(4 p+4)} S p(p)$-invariant as well which shows the assertion.

Consider $\left(G \times G^{\prime} \times V, g_{\varepsilon}+g^{\prime}+g_{V}\right)$ with $g_{\varepsilon}$ and $g_{V}$ defined in the proof of Theorem 2.1 and $g^{\prime}$ on $G^{\prime}$ biinvariant. Since $K \subset K^{\prime}$ is a normal subgroup, our hypothesis implies that $K^{\prime}$ acts on $V$ by elements of $\operatorname{Norm}_{O(V)} K$ and hence by isometries of $g_{V}$. Also, $g_{\varepsilon}+g^{\prime}$ is invariant under right multiplication of $G \times G^{\prime}$ by $K^{\prime} \subset \operatorname{Norm}_{G}(K) \times G^{\prime}$. Since $M^{\prime}=\left(G \times G^{\prime}\right) \times_{K^{\prime}} V$ is the corresponding quotient, and (2) guarantees that the horizontal planes of the submersion $\left(G \times G^{\prime} \times V, g_{\varepsilon}+g^{\prime}+g_{V}\right) \rightarrow M^{\prime}$ have nonnegative curvature, it follows that the induced metric on $M^{\prime}$ has the asserted properties.

In order to show that this applies to essentially trivial bundles, it remains to verify that $G \times V=(G \times L) \times{ }_{L} V$ satisfies the hypothesis (2) if $L \subset O(V)$ acts transitively on the unit sphere $S^{l} \subset V$. Indeed, the normal homogeneous metric on $S^{l}=L / H$ has positive curvature by $[\mathrm{Be}]$. Thus,

$$
\left|X_{\mathfrak{m}} \wedge Y_{\mathfrak{m}}\right| \leq C\left|\left[X_{\mathfrak{m}}, Y_{\mathfrak{m}}\right]\right|=C\left|[X, Y]_{\mathfrak{l}}\right| \leq C|[X, Y]|
$$

where $\frac{1}{4} C^{-1}>0$ is a lower curvature bound for $S^{l}$.

It remains to prove, for each of the bundles which are claimed to admit such metrics in Theorems 1.2]1.3]1.4] 1.5, that either hypothesis (2) is satisfied or Corollary 2.2 applies. The first three examples of Theorem 1.2 and the first example of Theorem 1.3 correspond to the following triples $H \subset K \subset G$ :

1. $\operatorname{Spin}_{ \pm}(7) \subset \operatorname{Spin}(8) \subset \operatorname{Spin}(p+9)$ for $p \in\{0,1,2\}$, where the first inclusion is by the spinor representation (cf. section 4 for details), and the second is the lift of the standard embedding $S O(8) \subset S O(p+9)$,

2. $G_{2} \subset \operatorname{Spin}(7) \subset \operatorname{Spin}(p+8)$ for $p \in\{0,1\}$, where the second inclusion is the lift of the standard embedding $S O(7) \subset S O(p+8)$,

3. $S U(3) \subset S U(4) \cong \operatorname{Spin}(6) \subset \operatorname{Spin}(7)$,

4. $\operatorname{Spin}(5) \cong S p(2) \subset \operatorname{Spin}(6) \cong S U(4) \subset S U(5)$.

That these triples satisfy hypothesis (2) was proven in [STa. In all cases, this was achieved by verifying that

$$
[\mathfrak{s}, \mathfrak{s}] \cap[\mathfrak{m}, \mathfrak{m}]=\{0\}
$$

which implies the hypothesis. 
The remaining examples of Theorems 1.2 and 1.3 now all are obtained by applying Corollary 2.2 to the above examples.

Likewise, the second and fourth examples in Theorem 1.5 follow by applying Corollary 2.2 to the first and third examples, respectively. The latter correspond to the triples:

1. $S U(2)_{1} \subset S O(4) \subset G_{2}$,

2. $S p(p) \subset S p(1) \cdot S p(p) \subset S p(p+1)$.

The first triple was verified in [STa]. The second triple satisfies the hypothesis because $S p(p+1) / S p(p)=S^{4 p+3}$ is a sphere whose normal homogeneous metric has positive curvature with lower bound, say, $4 \varepsilon$. Then it follows that $|[X, Y]| \geq \varepsilon|X \wedge Y|$ for all $X, Y \in \mathfrak{m} \oplus \mathfrak{s}$, and since $|X \wedge Y| \geq\left|X_{\mathfrak{m}} \wedge Y_{\mathfrak{m}}\right|$, the hypothesis of Theorem [2.1] is satisfied for $C:=\varepsilon^{-1}$.

The examples in Theorem 1.4 correspond to the triples:

1. $S U(2)_{1} \cdot S^{1} \subset S O(4) \subset G_{2}$,

2. $S^{1} \cdot S p(p) \subset S p(1) \cdot S p(p) \subset S p(p+1)$,

each of which is formed from a rank 4 example by enlarging $H$; this change obviously maintains condition (2).

\section{Necessary conditions for normal homogeneous col- lars}

For the remainder of this paper, we assume that $M=G \times_{K} V$ is endowed with a $G$-invariant nonnegatively curved metric such that there exists a principal $G$-orbit in $M$ which is totally geodesic and normal homogeneous, i.e., it is induced by an Ad-invariant inner product $Q$ on $\mathfrak{g}$. This is slightly weaker than assuming that $M$ has a normal homogeneous collar, but it will imply the same rigidity.

As in (11), we have the $Q$-orthogonal decomposition $\mathfrak{g}=\mathfrak{h} \oplus \mathfrak{p}=\mathfrak{h} \oplus \mathfrak{m} \oplus \mathfrak{s}$. The goal of this section is to prove:

Theorem 3.1 If $\mathfrak{m}_{1} \subset \mathfrak{m}$ is any non-trivial $A d_{H}$-irreducible subspace such that $\mathfrak{m}$ contains no irreducible factor equivalent to $\mathfrak{m}_{1}$, then there exists a constant $C>0$ such that for all $X=X_{\mathfrak{m}}+X_{\mathfrak{s}}, Y=Y_{\mathfrak{m}}+Y_{\mathfrak{s}} \in \mathfrak{m}_{1} \oplus \mathfrak{s}$ we have:

$$
\left|X_{\mathfrak{m}} \wedge Y_{\mathfrak{m}}\right| \leq C|[X, Y]| .
$$

When $K / H$ is isotropy irreducible, the choice $\mathfrak{m}_{1}=\mathfrak{m}$ yields a converse to Theorem 2.1. Evidently, (44) implies that for $X, Y \in \mathfrak{m}_{1} \oplus \mathfrak{s}$, we can have $[X, Y]=0$ only if $X_{\mathfrak{m}}, Y_{\mathfrak{m}}$ are linearly dependent. However, the converse implication is false in general; cf. Remark 5.9.

Towards proving this theorem, first notice that the disk bundle $D \subset M$ which is bounded by the totally geodesic principal orbit is totally convex and thus has the singular orbit $\Sigma \subset D$ 
as its soul, since $\Sigma$ is a closed submanifold equidistant from the boundary. We can choose a point $p \in \Sigma$ and a unit-speed normal geodesic $c:[0, l] \rightarrow M$ with $c(0)=p$ and $c(l) \in \partial D$ such that $K$ is the stabilizer of $p$ and $H$ is the stabilizer of $c(t)$ for all $t \in(0, l]$. For each $t \in[0, l]$, there is a self-adjoint map $\varphi_{t}: \mathfrak{p} \rightarrow \mathfrak{p}$ such that

$$
\left\langle X^{*}, Y^{*}\right\rangle_{c(t)}=Q\left(X, \varphi_{t} Y\right)
$$

for all $X, Y \in \mathfrak{p}$, where $X^{*}, Y^{*}$ denotes the action fields of $X, Y$. Each $\varphi_{t}$ is positive definite, with the exception that $\left.\varphi_{0}\right|_{\mathfrak{m}}=0$. Thus, $D \backslash \Sigma$ is $G$-equivariantly isometric to the warped product $\left((0, l] \times(G / H), d t^{2}+g_{\varphi_{t}}\right)$, where $\left\{g_{\varphi_{t}}\right\}$ is the family of homogeneous metrics on $G / H$ determined by $\left\{\varphi_{t}\right\}$. By assumption, $\varphi_{l}=I d$.

Lemma 3.2 For each $t \in[0, l],\left.\varphi_{t}\right|_{\mathfrak{s}}=I d$. In particular, the soul $\Sigma$ is normal homogeneous.

Proof. For each $X \in \mathfrak{p}$, the action field $X^{*}$ on $M$ is Killing, so its restriction to the geodesic $c$ is a Jacobi field, which we denote as $X_{t}$. Thus, $X \mapsto X_{t}$ is an identification of $\mathfrak{p}$ with a family of Jacobi fields along $c$. This family has the property that

$$
\left\langle X_{t}, Y_{t}^{\prime}\right\rangle=\left\langle X_{t}^{\prime}, Y_{t}\right\rangle \text { for all } X, Y \in \mathfrak{p}
$$

because these derivatives are determined by the second fundamental forms of the principle orbits. For any $X \in \mathfrak{p}$ such that $X_{t}$ is parallel, we must have that $X \in \mathfrak{s}$. This is because, for all $Y \in \mathfrak{m}$, we know that $Y_{0}=0$ and

$$
\frac{d}{d t}\left\langle X_{t}, Y_{t}\right\rangle=\left\langle X_{t}^{\prime}, Y_{t}\right\rangle+\left\langle X_{t}, Y_{t}^{\prime}\right\rangle=2\left\langle X_{t}^{\prime}, Y_{t}\right\rangle=0
$$

thus, $0=\left\langle X_{l}, Y_{l}\right\rangle=Q\left(X, \varphi_{l} Y\right)=Q(X, Y)$.

Conversely, we wish to show that each element of $\mathfrak{s}$ determines a parallel Jacobi field along $c$, which will complete the proof. By Perelman's Theorem $[\mathrm{P}]$, each $\bar{X}_{0} \in T_{p} \Sigma$ extends to a parallel Jacobi field, $\bar{X}_{t}$, along $c$, with $\bar{X}_{l}$ tangent to $\partial D$. There exists some $X \in \mathfrak{p}$ with $X_{l}=\bar{X}_{l}$. Since $\partial D$ is totally geodesic, we also have $X^{\prime}(l)=0=\bar{X}_{l}^{\prime}$, so the two Jacobi fiels must agree: $X_{t}=\bar{X}_{t}$ for all $t$. Since $X_{t}$ is parallel, we know from above that $X \in \mathfrak{s}$, and in fact $X$ is the unique vector in $\mathfrak{s}$ identified with $\bar{X}_{0}$ via the identification $\mathfrak{s} \cong T_{p} \Sigma$ given by action fields. Thus, for all $X \in \mathfrak{s}, X_{t}$ is a parallel Jacobi field.

Before we prove Theorem 3.1, we need the following

Lemma 3.3 Let $H \subset K \subset G$ be as above, and let $\mathfrak{m}_{1} \subset \mathfrak{m}$ be a linear subspace. Then there are polynomials $\lambda_{i}$ for $i=1,2,3$ with the following property. If $\varphi: \mathfrak{p} \rightarrow \mathfrak{p}$ is a positive definite $A d_{H}$-equivariant map with $\left.\varphi\right|_{\mathfrak{s}}=I d$ and $\left.\varphi\right|_{\mathfrak{m}_{1}}=(1-h)^{-1}$ Id for some $h \in(-\infty, 1)$, and if we let $\psi:=I d-\varphi^{-1}$, then for all $X=X_{\mathfrak{m}}+X_{\mathfrak{s}}, Y=Y_{\mathfrak{m}}+Y_{\mathfrak{s}} \in \mathfrak{m}_{1} \oplus \mathfrak{s}$ we have

$$
k^{\varphi}\left(X^{\prime}, Y^{\prime}\right) \leq \lambda_{1}(|\psi|)|[X, Y]|^{2}+\lambda_{2}(|\psi|)|[X, Y]| \cdot\left|X_{\mathfrak{m}} \wedge Y_{\mathfrak{m}}\right|+\lambda_{3}(|\psi|) h^{2}\left|X_{\mathfrak{m}} \wedge Y_{\mathfrak{m}}\right|^{2},
$$

where $X^{\prime}:=\varphi^{-1} X, Y^{\prime}:=\varphi^{-1} Y$ and $k^{\varphi}$ denotes the unnormalized curvature of $\left(G / H, g_{\varphi}\right)$. 
Proof. For the proof, we use the description of the unnormalized curvature established in [STa, Proposition 2.4]. There, $k(t)$ denotes the curvature of the metric $\varphi_{t}=(I d-t \psi)^{-1}$. We need this formula for $t=1$ which reads

$$
k^{\varphi}\left(X^{\prime}, Y^{\prime}\right)=\alpha+\beta+\gamma+\delta-\frac{3}{4}\left|D^{\mathfrak{p}}\right|_{g_{1}}^{2} \leq \alpha+\beta+\gamma+\delta,
$$

where

$$
\begin{aligned}
\alpha= & \left|[X, Y]^{\mathfrak{h}}\right|^{2}+\frac{1}{4}\left|[X, Y]^{\mathfrak{p}}\right|^{2} \\
\beta= & -\frac{3}{4}\langle\psi[X, Y],[X, Y]\rangle-\frac{3}{2}\left\langle[X, Y]^{\mathfrak{h}}, A\right\rangle \\
\gamma= & -\frac{3}{4}|\psi[X, Y]|^{2}+\frac{3}{2}\langle\psi[X, Y], A\rangle-\frac{3}{2}\left\langle[X, Y]^{\mathfrak{m}}, B\right\rangle+\frac{3}{4}\left|A^{\mathfrak{h}}\right|^{2} \\
\delta= & -\frac{3}{4}\left\langle\psi^{3}[X, Y],[X, Y]\right\rangle+\frac{3}{2}\left\langle\psi^{2}[X, Y], A\right\rangle-\frac{3}{2}\langle\psi[X, Y], B\rangle \\
& -\frac{3}{4}\langle\psi A, A\rangle-\frac{1}{4}\langle\psi C, C\rangle+\langle\psi[\psi X, X],[\psi Y, Y]\rangle+\langle A, B\rangle-\frac{3}{2}\left\langle A^{\mathfrak{h}}, B\right\rangle
\end{aligned}
$$

and

$$
\begin{aligned}
A & =[\psi X, Y]+[X, \psi Y]=h\left(2\left[X_{\mathfrak{m}}, Y_{\mathfrak{m}}\right]+\left[X_{\mathfrak{m}}, Y_{\mathfrak{s}}\right]+\left[X_{\mathfrak{s}}, Y_{\mathfrak{m}}\right]\right) \\
B & =[\psi X, \psi Y]=h^{2}\left[X_{\mathfrak{m}}, Y_{\mathfrak{m}}\right] \in \mathfrak{k}, \\
C & =[\psi X, Y]-[X, \psi Y]=h\left(\left[X_{\mathfrak{m}}, Y_{\mathfrak{s}}\right]-\left[X_{\mathfrak{s}}, Y_{\mathfrak{m}}\right]\right) \in \mathfrak{s} .
\end{aligned}
$$

Let us label the two norms

$$
N_{1}:=|[X, Y]| \text { and } N_{2}:=\left|X_{\mathfrak{m}} \wedge Y_{\mathfrak{m}}\right|
$$

There is a constant $\lambda>0$ such that for all $X_{\mathfrak{m}}, Y_{\mathfrak{m}} \in \mathfrak{m}_{1}$ we have

$$
\left|\left[X_{\mathfrak{m}}, Y_{\mathfrak{m}}\right]\right| \leq \lambda N_{2}
$$

where $\lambda$ is the norm of the linear map [, ] $: \Lambda^{2} \mathfrak{m}_{1} \rightarrow \mathfrak{k}$. Thus,

$$
\begin{aligned}
\left|A^{\mathfrak{h}}\right| \leq\left|A^{\mathfrak{k}}\right| & =2|h|\left|\left[X_{\mathfrak{m}}, Y_{\mathfrak{m}}\right]\right| \leq 2 \lambda|h| N_{2}, \\
|B| & \leq \lambda h^{2} N_{2} .
\end{aligned}
$$

Moreover, since $C \in \mathfrak{s}$ we have $\psi C=0$, and $[\psi X, X]=h\left[X_{\mathfrak{m}}, X_{\mathfrak{s}}\right] \in \mathfrak{s}$ so that $\psi[\psi X, X]=0$. Thus, using in addition that $|h| \leq|\psi|$, we obtain the following estimates.

$$
\begin{aligned}
& \alpha \leq N_{1}^{2} \\
& \beta \leq \frac{3}{4}|\psi| N_{1}^{2}+\frac{3}{2} N_{1}\left|A^{\mathfrak{h}}\right| \leq \frac{3}{4}|\psi| N_{1}^{2}+3 \lambda|h| N_{1} N_{2} \leq \frac{3}{4}|\psi| N_{1}^{2}+3 \lambda|\psi| N_{1} N_{2}
\end{aligned}
$$




$$
\begin{aligned}
\gamma \leq & \frac{3}{2} N_{1}\left|\psi A^{\mathfrak{k}}\right|+\frac{3}{2} N_{1}|B|+\frac{3}{4}\left|A^{\mathfrak{h}}\right|^{2} \\
\leq & 3 \lambda|h||\psi| N_{1} N_{2}+\frac{3}{2} \lambda h^{2} N_{1} N_{2}+3 \lambda^{2} h^{2} N_{2}^{2} \\
\leq & \frac{9}{2} \lambda|\psi|^{2} N_{1} N_{2}+3 \lambda^{2} h^{2} N_{2}^{2} \\
\delta \leq & \frac{3}{4}|\psi|^{3} N_{1}^{2}+\frac{3}{2} N_{1}\left|\psi^{2} A^{\mathfrak{k}}\right|+\frac{3}{2}|\psi| N_{1}|B|+\frac{3}{4}\left|\psi A^{\mathfrak{k}}\right|\left|A^{\mathfrak{k}}\right|+\frac{5}{2}\left|A^{\mathfrak{k}}\right||B| \\
\leq & \frac{3}{4}|\psi|^{3} N_{1}^{2}+\left(3 \lambda|h||\psi|^{2}+\frac{3}{2} \lambda h^{2}|\psi|\right) N_{1} N_{2} \\
& +\left(3 \lambda^{2} h^{2}|\psi|+5 \lambda^{2}|h|^{3}\right) N_{2}^{2} \\
\leq & \frac{3}{4}|\psi|^{3} N_{1}^{2}+\frac{9}{2} \lambda|\psi|^{3} N_{1} N_{2}+8 \lambda^{2} h^{2}|\psi| N_{2}^{2}
\end{aligned}
$$

In the estimate of $\gamma$, we dropped the first term as it is nonpositive. Substituting all of this into (5) we obtain

$$
\begin{aligned}
k^{\varphi}\left(X^{\prime}, Y^{\prime}\right) \leq & \left(1+\frac{3}{4}|\psi|+\frac{3}{4}|\psi|^{3}\right) N_{1}^{2}+\left(3 \lambda|\psi|+\frac{9}{2} \lambda|\psi|^{2}+\frac{9}{2} \lambda|\psi|^{3}\right) N_{1} N_{2} \\
& +\left(3 \lambda^{2}+8 \lambda^{2}|\psi|\right) h^{2} N_{2}^{2}
\end{aligned}
$$

which shows the claim.

We are now ready to prove Theorem 3.1 ,

Proof. Let $\mathfrak{m}_{1} \subset \mathfrak{m}$ be as in the theorem. By Schur's Lemma, $\left.\varphi_{t}\right|_{\mathfrak{m}_{1}}=f(t) \cdot I d$ for some smooth function $f:(0, l] \rightarrow \mathbb{R}^{+}$, and we let $\psi_{t}:=I d-\varphi_{t}^{-1}$. Thus, by Lemma 3.2, we have $\left.\varphi_{t}\right|_{\mathfrak{s}}=I d$ and $\left.\varphi_{t}\right|_{\mathfrak{m}_{1}}=(1-h(t))^{-1} I d$ for $h(t):=1-f(t)^{-1}$. Moreover, $\psi_{l}=0$ since $t=l$ corresponds to the normal homogeneous principal orbit. Identifying $M \backslash \Sigma \cong$ $\left((0, l] \times(G / H), d t^{2}+g_{\varphi_{t}}\right)$, the unnormalized curvature $k^{M}$ of the tangent plane in $M$ at $c(t)$ spanned by the action fields of $X^{\prime}$ and $Y^{\prime}$ equals:

$$
k^{M}\left(X^{\prime}, Y^{\prime}\right)=k^{\varphi_{t}}\left(X^{\prime}, Y^{\prime}\right)+I I\left(X^{\prime}, Y^{\prime}\right)^{2}-I I\left(X^{\prime}, X^{\prime}\right) I I\left(Y^{\prime}, Y^{\prime}\right) .
$$

The second fundamental form of this warped product metric satisfies:

$$
I I\left(X^{\prime}, Y^{\prime}\right)=\frac{1}{2} Q\left(X^{\prime}, \dot{\varphi}_{t} Y^{\prime}\right)=\frac{1}{2} h^{\prime}(t) Q\left(X_{\mathfrak{m}}, Y_{\mathfrak{m}}\right) .
$$

Thus, by Lemma 3.3 ,

$$
\begin{aligned}
k^{M}\left(X^{\prime}, Y^{\prime}\right)= & k^{\varphi_{t}}\left(X^{\prime}, Y^{\prime}\right)-\frac{1}{4} h^{\prime}(t)^{2}\left|X_{\mathfrak{m}} \wedge Y_{\mathfrak{m}}\right|^{2} \\
\leq & \lambda_{1}\left(\left|\psi_{t}\right|\right)|[X, Y]|^{2}+\lambda_{2}\left(\left|\psi_{t}\right|\right)|[X, Y]| \cdot\left|X_{\mathfrak{m}} \wedge Y_{\mathfrak{m}}\right| \\
& +\lambda_{3}\left(\left|\psi_{t}\right|\right) h(t)^{2}\left|X_{\mathfrak{m}} \wedge Y_{\mathfrak{m}}\right|^{2}-\frac{1}{4} h^{\prime}(t)^{2}\left|X_{\mathfrak{m}} \wedge Y_{\mathfrak{m}}\right|^{2} .
\end{aligned}
$$


Since we assume that the metric on $M$ has nonnegative curvature, this implies for all $X=$ $X_{\mathfrak{m}}+X_{\mathfrak{s}}, Y=Y_{\mathfrak{m}}+Y_{\mathfrak{s}} \in \mathfrak{m}_{1} \oplus \mathfrak{s}$ with $X_{\mathfrak{m}} \wedge Y_{\mathfrak{m}} \neq 0$

$$
\begin{aligned}
0 \leq \frac{k^{M}\left(X^{\prime}, Y^{\prime}\right)}{\left|X_{\mathfrak{m}} \wedge Y_{\mathfrak{m}}\right|^{2}} \leq & \lambda_{1}\left(\left|\psi_{t}\right|\right) \rho(X, Y)^{2}+\lambda_{2}\left(\left|\psi_{t}\right|\right) \rho(X, Y) \\
& +\lambda_{3}\left(\left|\psi_{t}\right|\right) h(t)^{2}-\frac{1}{4} h^{\prime}(t)^{2}
\end{aligned}
$$

where $\rho(X, Y):=\frac{|[X, Y]|}{\left|X_{\mathfrak{m}} \wedge Y_{\mathfrak{m}}\right|}$. Suppose the theorem is false, which means that

$$
\inf \left\{\rho(X, Y) \mid X=X_{\mathfrak{m}}+X_{\mathfrak{s}}, Y=Y_{\mathfrak{m}}+Y_{\mathfrak{s}} \in \mathfrak{m}_{1} \oplus \mathfrak{s}, X_{\mathfrak{m}} \wedge Y_{\mathfrak{m}} \neq 0\right\}=0 .
$$

Since (6) must hold for all $X, Y \in \mathfrak{m}_{1} \oplus \mathfrak{s}$ with $X_{\mathfrak{m}} \wedge Y_{\mathfrak{m}} \neq 0$, (7) implies

$$
0 \leq \lambda_{3}\left(\left|\psi_{t}\right|\right) h(t)^{2}-\frac{1}{4} h^{\prime}(t)^{2}
$$

Suppose that there is a $t_{0} \in(0, l]$ such that $h\left(t_{0}\right) \neq 0$, and let $t_{1}:=\min \left\{t \in\left(t_{0}, l\right] \mid h(t)=0\right\}$ which exists as $h(l)=0$. Since $\lambda_{3}$ is continuous, it follows that $\lambda_{3}\left(\left|\psi_{t}\right|\right) \leq C$ for all $t \in\left[t_{0}, t_{1}\right]$ and some constant $C>0$, and hence by (8),

$$
\left(\log (h(t))^{\prime}\right)^{2}=\frac{h^{\prime}(t)^{2}}{h(t)^{2}} \leq 4 \lambda_{3}\left(\left|\psi_{t}\right|\right) \leq 4 C
$$

for all $t \in\left[t_{0}, t_{1}\right)$, i.e., $\log (h(t))$ has bounded derivative for all such $t$. On the other hand, $\lim _{t \nearrow t_{1}} \log h(t)=-\infty$ which is impossible.

This shows that we must have $h(t) \equiv 0$ and hence $f(t) \equiv 1$ on $(0, l]$. Note that for all $X \in \mathfrak{m}$ we have $\lim _{t \rightarrow 0}\left(X_{\mathfrak{m}}\right)_{c(t)}=0$. Thus, for $X_{\mathfrak{m}} \in \mathfrak{m}_{1}$ we have $0=\lim _{t \rightarrow 0} g_{c(t)}\left(X_{\mathfrak{m}}, X_{\mathfrak{m}}\right)=$ $\lim _{t \rightarrow 0} Q\left(X_{\mathfrak{m}}, \varphi_{t} X_{\mathfrak{m}}\right)=\left|X_{\mathfrak{m}}\right|_{Q}^{2}$ which is a contradiction.

\section{Octonions and Triality}

In this section, we will collect some facts about the octonion numbers and triality which are well known (for a survey, see e.g. [Ba]), and show that certain triples $H \subset K \subset G$ do not satisfy condition (4) from Theorem 3.1.

Let $\mathbb{O} \cong \mathbb{R}^{8}$ denote the octonian numbers, and let $G_{2}$ be the automorphism group of $\mathbb{O}$. Since $G_{2}$ stabilizes $1 \in \mathbb{O}$ and is orthogonal, it leaves $\operatorname{Im}(\mathbb{O}) \cong \mathbb{R}^{7}$ invariant. We consider the $A d_{G_{2}}$-invariant decomposition

$$
\mathfrak{s o}(8)=\mathfrak{s o}(\mathbb{O})=\mathfrak{g}_{2} \oplus\left\{L_{q} \mid q \in \operatorname{Im}(\mathbb{O})\right\} \oplus\left\{R_{q} \mid q \in \operatorname{Im}(\mathbb{O})\right\}:=\mathfrak{g}_{2} \oplus V_{L} \oplus V_{R}
$$

where $L_{q}, R_{q}: \mathbb{O} \rightarrow \mathbb{O}$ denote multiplication from the left and the right, respectively. Indeed, $V_{L}, V_{R}$ both are $A d_{G_{2}}$-invariant and not equal so that their intersection must vanish. Then a dimension count shows that the three summands on the right of (9) span all of $\mathfrak{s o}(8)$. Note, however, that $V_{L}$ and $V_{R}$ are not orthogonal; in fact, these spaces intersect at an angle of $\pi / 3$. 
Let $\operatorname{Spin}_{0}(7) \subset \operatorname{Spin}(8)$ be the subgroup obtained by the lift of the inclusion $S O(7) \subset$ $S O(8)$ of endomorphisms stabilizing $1 \in \mathbb{O}$. Then all elements in its Lie algebra $\mathfrak{s o}_{0}(7) \subset$ $\mathfrak{s o}(8)$ vanish on 1 , hence we obtain the orthogonal decomposition

$$
\mathfrak{s o}(8)=\underbrace{\mathfrak{g}_{2} \oplus \mathfrak{m}_{0}}_{=\mathfrak{s o}_{0}(7)} \oplus \mathfrak{s}_{0}, \text { where } \begin{aligned}
\mathfrak{m}_{0} & =\left\{L_{q}-R_{q} \mid q \in \operatorname{Im}(\mathbb{O})\right\}, \text { and } \\
\mathfrak{s}_{0} & =\left\{L_{q}+R_{q} \mid q \in \operatorname{Im}(\mathbb{O})\right\} .
\end{aligned}
$$

As a $G_{2}$-module, the decomposition (9) can be written as

$$
\mathfrak{s o}(8)=\mathfrak{g}_{2} \oplus\left(\mathbb{R}^{2} \otimes(\operatorname{Im}(\mathbb{O}))\right),
$$

hence any automorphism of $\operatorname{Spin}(8)$ which leaves $G_{2}$ invariant determines an element of $O(2)$ acting on the $\mathbb{R}^{2}$-factor.

The triality group is defined as the group of outer automorphisms of $\operatorname{Spin}(8)$. This group is isomorphic to the permutation group $S_{3}$. Moreover, each outer automorphism can be uniquely represented such that it acts on $G_{2} \subset \operatorname{Spin}(8)$ as the identity. Thus, by the preceding paragraph, the triality automorphisms induce a faithful homomorphism $S_{3} \rightarrow O(2)$ and hence, $S_{3}$ acts on $\mathbb{R}^{2}$ as the isometry group of an equilateral triangle. Therefore, the orbit of $\operatorname{Spin}_{0}(7) \subset \operatorname{Spin}(8)$ under the triality group consists of three subgroups

$$
\operatorname{Spin}_{0}(7), \operatorname{Spin}_{ \pm}(7) \hookrightarrow \operatorname{Spin}(8)
$$

and the Lie algebras $\mathfrak{s o}_{ \pm}(7) \subset \mathfrak{s o}(8)$ of the latter induce the orthogonal decompositions

$$
\mathfrak{s o}(8)=\underbrace{\mathfrak{g}_{2} \oplus \mathfrak{m}_{ \pm}}_{=\mathfrak{s o}_{ \pm}(7)} \oplus \mathfrak{s}_{ \pm}, \text {where } \begin{aligned}
& \mathfrak{m}_{+}=\left\{L_{q}+2 R_{q} \mid q \in \operatorname{Im}(\mathbb{O})\right\}, \\
& \mathfrak{m}_{-}=\left\{2 L_{q}+R_{q} \mid q \in \operatorname{Im}(\mathbb{O})\right\},
\end{aligned} \quad \text { and } \begin{aligned}
& \mathfrak{s}_{+}=V_{L}, \\
& \mathfrak{s}_{-}=V_{R} .
\end{aligned}
$$

Let $\operatorname{Spin}(8) \subset \operatorname{Spin}(9)$ be the lift of the inclusion $S O(8) \subset S O(9)$. Then $\operatorname{Spin}_{0}(7) \subset$ $\operatorname{Spin}(8) \subset \operatorname{Spin}(9)$ is the lift of the inclusion $S O(7) \subset S O(9)$, whereas $\operatorname{Spin}_{ \pm}(7) \subset \operatorname{Spin}(8) \subset$ $\operatorname{Spin}(9)$ are conjugate subgroups which are the isotropy of $S^{15}=\operatorname{Spin}(9) / \operatorname{Spin}(7)$.

Proposition 4.1 For the following triples $H \subset K \subset G$, condition (4) of Theorem 3.1 with $\mathfrak{m}_{1}=\mathfrak{m}$ is violated.

1. $G_{2} \subset \operatorname{Spin}_{ \pm}(7) \subset \operatorname{Spin}(p+9)$ for $p \geq 0$,

2. $G_{2} \subset \operatorname{Spin}_{0}(7) \subset \operatorname{Spin}(p+9)$ for $p \geq 1$,

3. $G_{2} \subset \operatorname{Spin}_{0}(7) \subset F_{4}$,

4. $\operatorname{Spin}_{ \pm}(7) \subset \operatorname{Spin}(8) \subset \operatorname{Spin}(p+9)$ for $p \geq 3$,

where the second inclusions are given by the composition of the inclusion $\operatorname{Spin}_{i}(7) \subset \operatorname{Spin}(9)$ from above with the lift of the inclusion $S O(9) \subset S O(p+9)$ in the first tow cases and with the isotropy of the Cayley plane $F_{4} / \operatorname{Spin}(9)$ in the third case, and by the lift of $S O(8) \subset S O(p+9)$ in the last case. 
Proof. Decompose $\mathbb{R}^{p+9}=\mathbb{O} \oplus \mathbb{R}^{p+1}$. In the first two cases, we have the splitting

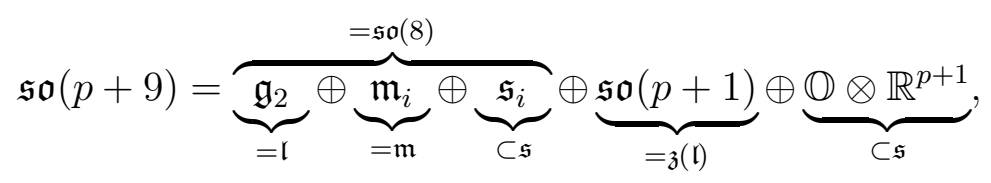

where $\operatorname{Spin}(8) \times \operatorname{Spin}(p+1)$ acts on $\mathbb{O} \otimes \mathbb{R}^{p+1}$ by the tensor representation of $S O(8) \times$ $S O(p+1)$.

To show the assertion for the first triple, pick the orthonormal set $e_{1}=1, e_{2}=i, e_{3}=$ $j \in \mathbb{O}$ and $e_{4} \in \mathbb{R}^{p+1}$. Moreover, let $E_{r s}=-E_{s r} \in \mathfrak{s o}(8+p)$ denote the rank two matrix with $E_{r s} e_{r}=e_{s}$ and $E_{r s} e_{s}=-e_{r}$, and define

$$
X:=E_{12}+E_{34}, \text { and } Y:=E_{13}+E_{24} .
$$

Then $[X, Y]=0$, and $E_{24}, E_{34} \in \mathbb{O} \otimes \mathbb{R}^{p+1} \subset \mathfrak{s}$. Moreover, $2 E_{12}=L_{i}+R_{i}, 2 E_{13}=L_{j}+R_{j} \in$ $\mathfrak{s}_{0}$, so that $X, Y \in \mathfrak{g}_{2}^{\perp}$. If the $\mathfrak{m}_{ \pm}$-components of $X$ and $Y$ were linearly dependent, then the span of $E_{12}, E_{13} \in \mathfrak{s}_{0}$ would intersect $\mathfrak{s}_{ \pm}$which is impossible. Thus, $X_{\mathfrak{m}_{ \pm}}, Y_{\mathfrak{m}_{ \pm}}$are linearly independent which shows that (4) does not hold.

For the second triple, we write $\mathbb{O}=\mathbb{H} \oplus \varepsilon \mathbb{H}$ where $\mathbb{H}$ denote the quaternions. Pick an orthonormal basis $e_{1}, \ldots, e_{4}$ of $\varepsilon \mathbb{H}$ by

$$
e_{1}:=\varepsilon, e_{2}:=\varepsilon k, e_{3}:=\varepsilon i, e_{4}:=\varepsilon j,
$$

and let $e_{5}, e_{6} \in \mathbb{R}^{p+1}$ be othonormal. Let $L_{i}, R_{j} \in \mathfrak{s o}(7)$ be defined as above. Then $\left[L_{i}, R_{j}\right] \in$ $\mathfrak{s o}(7)$ is the associator, whence $\left.\left[L_{i}, R_{j}\right]\right|_{\mathbb{H}}=0$ whereas $\left.\left[L_{i}, R_{j}\right]\right|_{\varepsilon \cdot \mathbb{H}}=\left.2 L_{k}\right|_{\varepsilon \cdot \mathbb{H}}$. Thus, in the matrix notation from above, we may write w.r.t. the above basis

$$
\left[L_{i}, R_{j}\right]=-2\left(E_{12}+E_{34}\right)
$$

Moreover, $L_{i} e_{2}=R_{j} e_{1}$ and $L_{i} e_{4}=R_{j} e_{3}$ so that $\left[L_{i}, E_{2 r}\right]=\left[R_{j}, E_{1 r}\right]$ and $\left[L_{i}, E_{4 r}\right]=\left[R_{j}, E_{3 r}\right]$ for $r=5,6$. Thus, if we define

$$
X:=L_{i}+\sqrt{2}\left(E_{15}+E_{36}\right) \text { and } Y:=R_{j}+\sqrt{2}\left(E_{25}+E_{46}\right) \in \mathfrak{m} \oplus \mathfrak{s},
$$

then one verifies from here that $[X, Y]=0$. But from (10) it follows that $X_{\mathfrak{m}}=\left(L_{i}\right)_{\mathfrak{m}}=$ $\frac{1}{2}\left(L_{i}-R_{i}\right)$ and $Y_{\mathfrak{m}}=\left(R_{j}\right)_{\mathfrak{m}}=-\frac{1}{2}\left(L_{j}-R_{j}\right)$, i.e., $X_{\mathfrak{m}}, Y_{\mathfrak{m}}$ are linearly independent which shows that (4) does not hold.

Now let us show that the third triple does not satisfy (44). The Lie algebra $\mathfrak{f}_{4}$ of $F_{4}$ can be decomposed as

$$
\mathfrak{f}_{4}=\mathfrak{s o}(8) \oplus V_{8} \oplus \Delta_{8}^{+} \oplus \Delta_{8}^{-},
$$

where $V_{8}$ is the standard and $\Delta_{8}^{ \pm}$are the spin representations of $\mathfrak{s o}(8)$. Moreover, $\mathfrak{s o}(8) \oplus V_{8}:=$ $\mathfrak{s o}_{0}(9) \subset \mathfrak{f}_{4}$ and $\mathfrak{s o}(8) \oplus \Delta_{8}^{ \pm}=: \mathfrak{s o}_{ \pm}(9) \subset \mathfrak{f}_{4}$ are Lie subalgebras corresponding to conjugate Lie subgroups $\operatorname{Spin}_{i}(9) \subset F_{4}$ with $i \in\{0, \pm\}$ whose intersection is $\operatorname{Spin}(8)$.

In particular, the inclusions $\operatorname{Spin}_{0}(7) \subset \operatorname{Spin}(8) \subset \operatorname{Spin}_{ \pm}(9)$ correspond to the isotropy inclusion of $S^{15}=\operatorname{Spin}(9) / \operatorname{Spin}(7)$ and hence, the triples $G_{2} \subset \operatorname{Spin}_{0}(7) \subset \operatorname{Spin}_{ \pm}(9)$ do not 
satisfy (4) by the first case considered above, and hence, when enlarging $\operatorname{Spin}_{ \pm}(9)$ to $F_{4}$, (4) remains violated.

Finally, for the last example, it suffices to consider $\operatorname{Spin}_{+}(7) \subset \operatorname{Spin}(8) \subset \operatorname{Spin}(p+9)$, so that $\mathfrak{m}=\mathfrak{s o}(8) \cap \mathfrak{s o}_{+}(7)^{\perp}=\mathfrak{s}_{+}=V_{L}$. We consider an orthonormal set of $\mathbb{R}^{p+9}=\mathbb{O} \oplus \mathbb{R}^{p+1}$ as

$e_{1}:=1, e_{2}:=k, e_{3}:=i, e_{4}:=j$, and $e_{4+r}=\varepsilon e_{r}$ for $r=1, \ldots, 4$, and $e_{9}, \ldots, e_{12} \in \mathbb{R}^{p+1}$.

Note that $\left.\left[L_{i}, L_{j}\right]\right|_{\mathbb{H}}=2 L_{k}$, whereas $\left.\left[L_{i}, L_{j}\right]\right|_{\mathbb{H} \varepsilon}=-2 L_{k}$. Thus, in the notation from above, we have $\left[L_{i}, L_{j}\right]=2\left(E_{12}+E_{34}+E_{56}+E_{78}\right)$. Moreover, $L_{i} e_{2 r-1}=L_{j} e_{2 r}$ so that $\left[L_{i}, E_{2 r-1, s}\right]=\left[L_{j}, E_{2 r, s}\right]$ for $r=1, \ldots, 4$ and $s \geq 9$. Thus, for the elements $X, Y \in \mathfrak{m} \oplus \mathfrak{s}$ given as

$$
X=L_{i}+\sqrt{2} \sum_{r=1}^{4} E_{2 r, 8+r} \text { and } Y=L_{j}+\sqrt{2} \sum_{r=1}^{4} E_{2 r-1,8+r},
$$

one verifies that $[X, Y]=0$ whereas $X_{\mathfrak{m}}=L_{i}$ and $Y_{\mathfrak{m}}=L_{j}$ are linearly independent which contradicts (41).

\section{Bundles with normal homogeneous collar}

In this final section, we derive consequences of Theorem 3.1, and give a partial classification of the triples $H \subset K \subset G$ for which condition (4) can hold.

We let $\mathfrak{k}_{0}:=\langle\mathfrak{m}\rangle \triangleleft \mathfrak{k}$ be the ideal generated by $\mathfrak{m}$ and let $\mathfrak{h}_{0}:=\mathfrak{h} \cap \mathfrak{k}_{0}$, so that $K_{0} / H_{0}$ is an almost effective sphere where $H_{0} \subset H$ and $K_{0} \subset K$ are the connected normal subgroups with Lie algebra $\mathfrak{h}_{0}$ and $\mathfrak{k}_{0}$, respectively. If we let $\mathfrak{h}^{\prime} \triangleleft \mathfrak{h}$ be the ineffective kernel of this action, then we obtain the $Q$-orthogonal decompositions

$$
\mathfrak{h}=\mathfrak{h}^{\prime} \oplus \mathfrak{h}_{0}, \quad \mathfrak{k}_{0}=\mathfrak{h}_{0} \oplus \mathfrak{m}, \quad \mathfrak{k}=\mathfrak{h}^{\prime} \oplus \mathfrak{k}_{0} .
$$

The almost effective transitive actions on spheres have been classified ([MS]). For each of these actions, we pick a subgroup $H_{1} \subset K_{0}$ which contains $H_{0}$. Namely, for the homogeneous sphere $S^{15}=\operatorname{Spin}(9) / \operatorname{Spin}(7)$, we let $H_{1}:=\operatorname{Spin}(8)$, whereas in all other cases, we let $H_{1}:=\left(\text { Norm }_{K_{0}} H_{0}\right)_{0}$ be the identity component of the normailzer of $H_{0} \subset K_{0}$. We denote the corresponding $Q$-orthogonal decomposition by

$$
\mathfrak{k}_{0}=\mathfrak{h}_{1} \oplus \mathfrak{m}_{1}
$$

From the classification in [MS] it follows that either $H_{0}=1$ and $H_{1}=K_{0}$, so that $\mathfrak{m}_{1}=0$, which happens only for $S^{1}=U(1) / 1$ or $S^{3}=S U(2) / 1$, or the representation of $H_{1}$ on $\mathfrak{m}_{1}:=\mathfrak{h}_{1}^{\perp}$ is irreducible and there is no irreducible $A d_{H}$-module in $\mathfrak{m}$ which is equivalent to $\mathfrak{m}_{1} \subset \mathfrak{m}$, so that we can choose this particular space for condition (4) in Theorem 3.1 .

We shall from now on assume that $\mathfrak{m}_{1} \neq 0$, thus assuming that $\operatorname{dim} H_{0}>0$ and hence, $\operatorname{dim}(K / H) \geq 2$. Moreover, we let $\mathfrak{l}:=\left\langle\mathfrak{m}_{1}\right\rangle \subset \mathfrak{k}_{0}$ be the Lie algebra generated by $\mathfrak{m}_{1}$, and let 
Table 1

Almost effective transitive aCtions of CONnECted Lie Groups on SPHeres $S^{n}=K_{0} / H_{0}$ WITH $\operatorname{dim} H_{0}>0$

\begin{tabular}{|c|c|c|c|c|c|c|}
\hline & $\operatorname{dim} S^{n}$ & $K_{0}$ & $H_{0}$ & $H_{1}$ & $\mathfrak{m}_{1}$ & $L$ \\
\hline 1 & $n \geq 2$ & $\begin{array}{c}S O(n+1) \\
\operatorname{Spin}(n+1)\end{array}$ & $\begin{array}{c}S O(n) \\
\operatorname{Spin}(n)\end{array}$ & $\begin{array}{c}S O(n) \\
\operatorname{Spin}(n)\end{array}$ & $\mathbb{R}^{n}$ & $\begin{array}{c}S O(n+1) \\
\operatorname{Spin}(n+1)\end{array}$ \\
\hline 2 & $\begin{array}{c}2 m+1 \\
m \geq 2\end{array}$ & $\begin{array}{c}T \cdot S U(m+1), \\
T \subset S^{1} \text { connected }\end{array}$ & $T \cdot S U(m)$ & $T \cdot U(m)$ & $\mathbb{C}^{m}$ & $S U(m+1)$ \\
\hline 3 & $\begin{array}{c}4 m+3 \\
m \geq 1\end{array}$ & $\begin{array}{c}T \cdot S p(m+1), \\
C \operatorname{Sp}(1) \text { connected }\end{array}$ & $T \cdot S p(m)$ & $T \cdot S p(1) \cdot S p(m)$ & $\mathbb{H}^{m}$ & $S p(m+1)$ \\
\hline 7 & 6 & $G_{2}$ & $S U(3)$ & $S U(3)$ & $\mathbb{C}^{3}$ & $G_{2}$ \\
\hline 8 & 7 & $S p i n(7)$ & $G_{2}$ & $G_{2}$ & $\mathbb{R}^{7}$ & $\operatorname{Spin}(7)$ \\
\hline 9 & 15 & $\operatorname{Spin}(9)$ & $S p i n(7)$ & $S p i n(8)$ & $\mathbb{R}^{8}$ & $\operatorname{Spin}(9)$ \\
\hline
\end{tabular}

The representations of $H_{1}$ on $\mathfrak{m}_{1}$ are the standard irreducible representations in each case.

$L \subset K_{0}$ be the corresponding connected subgroup. In Table 1, we now list all these groups which follow from the classification in [MS].

Proposition 5.1 Let $H \subset K \subset G$ and $\mathfrak{h} \subset \mathfrak{k} \subset \mathfrak{g}$ and $\mathfrak{m}_{1} \subset \mathfrak{m}$ be as above such that (4) is satisfied. Suppose there are elements $0 \neq X_{\mathfrak{m}} \in \mathfrak{m}_{1}$ and $0 \neq Y_{\mathfrak{s}} \in \mathfrak{s}$ such that $\left[X_{\mathfrak{m}}, Y_{\mathfrak{s}}\right]=0$. Let $N \subset K_{0}$ be the identity component of the centralizer of $Y_{s}$. Then $N \subset K_{0}$ acts transitively on $K_{0} / H_{1}$.

Proof. Evidently $N$ is closed and hence compact. Thus, $N$ acts transitively on $K_{0} / H_{1}$ if and only if $\operatorname{dim}\left(N /\left(N \cap H_{1}\right)\right)=\operatorname{dim}\left(K_{0} / H_{1}\right)$, since the former is the dimension of the $N$-orbit of $e H_{1} \in K_{0} / H_{1}$. Now this equation is equivalent to saying that the projection of $\mathfrak{n} \subset \mathfrak{k}_{0}$ to $\mathfrak{m}_{1}$ w.r.t. the splitting (12) is surjective, or, equivalently, that $\mathfrak{n}^{\perp} \cap \mathfrak{m}_{1}=0$, where $\mathfrak{n}=\mathfrak{z}\left(Y_{\mathfrak{s}}\right) \cap \mathfrak{k}_{0}$ is the Lie algebra of $N$.

Observe that $\mathfrak{n}^{\perp}=\mathfrak{z}\left(Y_{\mathfrak{s}}\right)^{\perp}+\mathfrak{k}_{0}^{\perp}=\left[Y_{\mathfrak{s}}, \mathfrak{g}\right]+\mathfrak{s}+\mathfrak{h}^{\prime}$. Thus, what we need to show is the following:

If for some $A \in \mathfrak{g}$ we have $\left[Y_{\mathfrak{s}}, A\right] \in \mathfrak{m}_{1} \oplus \mathfrak{s} \oplus \mathfrak{h}^{\prime}$, then $\left[Y_{\mathfrak{s}}, A\right] \in \mathfrak{s} \oplus \mathfrak{h}^{\prime}$.

Note that $\left[Y_{\mathfrak{s}}, \mathfrak{k}\right] \subset \mathfrak{s}$, so that it suffices to show (13) for all $A \in \mathfrak{s}$. Suppose therefore that for some $A \in \mathfrak{s}$ we have $\left[Y_{\mathfrak{s}}, A\right] \in \mathfrak{m}_{1} \oplus \mathfrak{s} \oplus \mathfrak{h}^{\prime}$.

Then, for $c \in \mathbb{R}$ we let $X:=X_{\mathfrak{m}}+c\left[X_{\mathfrak{m}}, A\right]$ and $Y:=Y_{\mathfrak{s}}+c\left[Y_{\mathfrak{s}}, A\right]_{\mathfrak{m}_{1} \oplus \mathfrak{s}}$. Since $\left[X_{\mathfrak{m}}, A\right] \in$ $[\mathfrak{k}, \mathfrak{s}] \subset \mathfrak{s}$, the $\mathfrak{m}_{1}$-component of $X$ is indeed $X_{\mathfrak{m}}$, and $X, Y \in \mathfrak{m}_{1} \oplus \mathfrak{s}$. Also, the $\mathfrak{m}_{1}$-component of $Y$ equals the $\mathfrak{m}_{1}$-component of $c\left[Y_{\mathfrak{s}}, A\right]$. Moreover, $Q\left(X_{\mathfrak{m}},\left[Y_{\mathfrak{s}}, A\right]\right)=Q\left(\left[X_{\mathfrak{m}}, Y_{\mathfrak{s}}\right], A\right)=0$ since $\left[X_{\mathfrak{m}}, Y_{\mathfrak{s}}\right]=0$, so that

$$
\left|X_{\mathfrak{m}} \wedge Y_{\mathfrak{m}}\right|_{Q}=|c|\left|X_{\mathfrak{m}} \wedge\left[Y_{\mathfrak{s}}, A\right]_{\mathfrak{m}_{1}}\right|_{Q}=|c|\left|X_{\mathfrak{m}}\right|_{Q}\left|\left[Y_{\mathfrak{s}}, A\right]_{\mathfrak{m}_{1}}\right|_{Q}
$$


On the other hand,

$$
\begin{aligned}
{[X, Y] } & =c(\left[X_{\mathfrak{m}},\left[Y_{\mathfrak{s}}, A\right]_{\mathfrak{m}_{1} \oplus \mathfrak{s}}\right]-\underbrace{\left[Y_{\mathfrak{s}},\left[X_{\mathfrak{m}}, A\right]\right]}_{=\left[X_{\mathfrak{m}},\left[Y_{\mathfrak{s}}, A\right]\right]})+c^{2}\left[\left[X_{\mathfrak{m}}, A\right],\left[Y_{\mathfrak{s}}, A\right]_{\mathfrak{m}_{1} \oplus \mathfrak{s}}\right] \\
& =-c\left[X_{\mathfrak{m}},\left[Y_{\mathfrak{s}}, A\right]_{\mathfrak{h}^{\prime}}\right]+c^{2}\left[\left[X_{\mathfrak{m}}, A\right],\left[Y_{\mathfrak{s}}, A\right]_{\mathfrak{m}_{1} \oplus \mathfrak{s}}\right] \\
& =c^{2}\left[\left[X_{\mathfrak{m}}, A\right],\left[Y_{\mathfrak{s}}, A\right]_{\mathfrak{m}_{1} \oplus \mathfrak{s}}\right]
\end{aligned}
$$

where the last equation follows since $\left[X_{\mathfrak{m}},\left[Y_{\mathfrak{s}}, A\right]_{\mathfrak{h}^{\prime}}\right] \in\left[\mathfrak{k}_{0}, \mathfrak{h}^{\prime}\right]=0$. Thus, by (4) we conclude that there is a $C>0$ such that for all $c \in \mathbb{R}$,

$$
|c|\left|X_{\mathfrak{m}}\right|_{Q}\left|\left[Y_{\mathfrak{s}}, A\right]_{\mathfrak{m}_{1}}\right|_{Q} \leq c^{2} C\left|\left[\left[X_{\mathfrak{m}}, A\right],\left[Y_{\mathfrak{s}}, A\right]_{\mathfrak{m}_{1} \oplus \mathfrak{s}}\right]\right|
$$

Dividing by $|c|$ and taking the limit for $c \rightarrow 0$, we conclude that $\left[Y_{\mathfrak{s}}, A\right]_{\mathfrak{m}_{1}}=0$, i.e., $\left[Y_{\mathfrak{s}}, A\right] \in$ $\mathfrak{s} \oplus \mathfrak{h}^{\prime}$ which shows (13).

Let $H \subset K \subset G$ be as in Proposition 5.1 with $\operatorname{dim} H_{0}>0$. Let $L \subset K_{0}$ be the connected normal subgroup with Lie algebra $\mathfrak{l}=\left\langle\mathfrak{m}_{1}\right\rangle \triangleleft \mathfrak{k}_{0}$ from Table 1 . We fix the following $Q_{-}$ orthogonal $A d_{L}$-invariant decomposition:

$$
\mathfrak{g}=\mathfrak{l} \oplus \mathfrak{z}(\mathfrak{l}) \oplus \bigoplus_{\alpha \in \Phi_{1}} V_{\alpha} \oplus \bigoplus_{\alpha \in \Phi_{2}} V_{\alpha}=: \mathfrak{n}(\mathfrak{l}) \oplus \mathfrak{s}_{1} \oplus \mathfrak{s}_{2},
$$

where $\mathfrak{z}(\mathfrak{l})$ and $\mathfrak{n}(\mathfrak{l})$ denote the centralizer and the normalizer of $\mathfrak{l}$, respectively, and $V_{\alpha}$ are non-trivial $A d_{L}$-irreducible subspaces for $\alpha \in \Phi:=\Phi_{1} \cup \Phi_{2}$, where

$\alpha \in \Phi_{1}$ if there are elements $0 \neq X_{\mathfrak{m}} \in \mathfrak{m}_{1}$ and $0 \neq Y_{s} \in V_{\alpha} \subset \mathfrak{s}$ such that $\left[X_{\mathfrak{m}}, Y_{\mathfrak{s}}\right]=0$

$\alpha \in \Phi_{2}$ otherwise.

Proposition 5.2 Let $H \subset K \subset G$ and $L \subset K_{0}$ be as above, i.e., $K_{0} / H_{0}$ is one of the entries in Table 1 and (4) holds, and consider the decomposition of $\mathfrak{g}$ from (14). Let $\alpha \in \Phi_{1}$. Then one of the following holds.

1. $L=K_{0}=S U(2) \cdot S U(2)^{\prime}$ so that $\mathfrak{k}_{0}=\mathfrak{s o}(4) \cong \mathfrak{s u}(2) \oplus \mathfrak{s u}(2)^{\prime}$, and $V_{\alpha}$ is odd dimensional. Furthermore, after permuting $\mathfrak{s u}(2)$ and $\mathfrak{s u}(2)^{\prime}$ if necessary, we have $\left[\mathfrak{s u}(2)^{\prime}, \mathfrak{s}_{1}\right]=0$.

2. $K_{0} / H_{0} \cong(T \cdot S U(4)) /(T \cdot S U(3))$ so that $L=S U(4)$, and $V_{\alpha}$ is the six-dimensional standard representation of $S O(6)=S U(4) / \mathbb{Z}_{2}$.

3. $K_{0} / H_{0} \cong \operatorname{Spin}(7) / G_{2}$ so that $L=\operatorname{Spin}(7)$, and $V_{\alpha}$ is the seven-dimensional standard representation of $S O(7)=\operatorname{Spin}(7) / \mathbb{Z}_{2}$.

Proof. Let $N \subset K_{0}$ be the stabilizer of $Y_{\mathfrak{s}} \in V_{\alpha}$ and $\mathfrak{n} \subset \mathfrak{k}_{0}$ be its Lie algebra. If $L \subset N$, then $A d_{L}\left(Y_{\mathfrak{s}}\right)=Y_{\mathfrak{s}}$, so that $Y_{\mathfrak{s}} \in \mathfrak{z}(\mathfrak{l})$ which is impossible. Thus, $L \not \subset N$.

By Proposition 5.1, $N \subset K_{0}$ acts transitively on $K_{0} / H_{1}$. We shall work through the possibilities for $K_{0}$ from Table 1. 
1. $K_{0} / H_{1}=S O(n+1) / S O(n)=S^{n}\left(\operatorname{Spin}(n+1) / \operatorname{Spin}(n)=S^{n}\right.$, resp. $) L=S O(n+1)$ $(L=\operatorname{Spin}(n+1)$, resp.).

In this case, $N \subsetneq S O(n+1)$ must be a subgroup which acts transitively on $S^{n}$, i.e., $N$ must be one of the entries of Table 1 . Also, any element $0 \neq X_{\mathfrak{m}} \in \mathfrak{m}_{1}=\mathfrak{m} \subset \mathfrak{s o}(m+1)$ is a matrix of real rank two, i.e., the Lie algebra $\mathfrak{n} \subset \mathfrak{s o}(n+1)$ of $N \subset S O(n+1)$ must contain such a matrix.

We claim that the only subgroup $N \subsetneq S O(n+1)$ from Table 1 whose Lie algebra contains elements of real rank two is $U(m) \subset S O(2 m)$. Namely, $\mathfrak{s u}(m) \subset \mathfrak{s o}(2 m)$ contains no elements of complex rank one and hence of real rank two. Next, any element of $\mathfrak{t} \oplus \mathfrak{s p}(m) \subset \mathfrak{s o}(4 m)$ for $m \geq 2$ is conjugate to an element of the form $X_{\mathfrak{m}}=\left(\lambda_{0} i, i \operatorname{diag}\left(\lambda_{1}, \ldots, \lambda_{m}\right)\right)$ with $\lambda_{i} \in \mathbb{R}$. Now the real rank of this element viewed as an endomorphism of $\mathbb{H}^{m}$ is easily seen to be at least 4, excluding this case. Next, since $\mathfrak{s u}(3) \subset \mathfrak{g}_{2}$ have equal rank, any element of $\mathfrak{g}_{2}$ is conjugate to an element of $\mathfrak{s u}(3) \subset \mathfrak{s o}(6) \subset \mathfrak{s o}(7)$. Since $\mathfrak{s u}(3)$ contains no matrices of real rank two, $\mathfrak{n} \cong \mathfrak{g}_{2}$ is also impossible. Likewise, if $X \in \mathfrak{s p i n}(7) \subset \mathfrak{s o}(8)$ had real rank two, then $X$ would lie in the isotropy algebra of $S^{7}=\operatorname{Spin}(7) / G_{2}$, hence $X \in \mathfrak{g}_{2} \subset \mathfrak{s p i n}(7)$ and thus cannot have rank two by the previous case. Finally, if $X \in \mathfrak{s p i n}(9)$ has rank two, then it lies in the isotropy algebra of $S^{15}=\operatorname{Spin}(9) / \operatorname{Spin}(7)$, acting on $\mathbb{R}^{7} \oplus \mathbb{R}^{8}$ via the standard and the spin representation, respectively. But by the previous, the action of $X \in \mathfrak{s p i n}(7)$ on $\mathbb{R}^{8}$ must have rank larger than two which rules out this case as well.

Let $T^{m} \subset S O(2 m)$ be a maximal torus with Lie algebra $\mathfrak{t}^{m} \subset \mathfrak{s o}(2 m)$, and suppose w.l.o.g. that $X_{\mathfrak{m}} \in \mathfrak{t}^{m}$. Let $V_{0} \subset \mathfrak{s}_{1}=\bigoplus_{\alpha \in \Phi_{1}} V_{\alpha}$ be the subspace stabilized by $T^{m}$. By our assumption, there are elements of $V_{\alpha}$ whose stabilizer is isomorphic to $U(m)$ and hence contains a maximal torus, so that $V_{0} \neq 0$. Since $X_{\mathfrak{m}} \in \mathfrak{t}^{m}$, it follows that the stabilizer of any $0 \neq Y \in V_{0}$ is conjugate $U(m)$ where $T^{m} \subset U(m) \subset S O(2 m)$. But there are only finitely many conjugates of $U(m)$ which contain $T^{m}$, namely the conjugates by elements of the Weyl group $W:=\operatorname{Norm}_{S O(2 m)} T^{m} / T^{m}$, so there are only finitely many choices for this stabilizer. On the other hand, the stabilizer of $0 \neq Y \in V_{0}$ depends continuously on $Y$, hence all of $V_{0}$ is stabilized by a fixed subgroup $U(m) \subset S O(2 m)$.

Since $V_{0}$ is invariant under the action of the Weyl group $W$, it follows that $U(m)$ is invariant under conjugation by $W$ as well. In particular $\mathfrak{z}(\mathfrak{u}(m)) \subset \mathfrak{t}^{m}$ is invariant under the Weyl group, i.e., the Weyl group cannot act irreducibly on $\mathfrak{t}^{m}$, hence $S O(2 m)$ cannot be simple, so that $m=2$, i.e., $N=U(2) \subset S O(4)$.

In particular, $S U(2) \subset N$ acts trivially on $V_{0}$, and since $S U(2) \subset S O(4)$ is a normal subgroup, it must act trivially on the invariant subspace generated by $V_{0}$ which is all of $\mathfrak{s}_{1}$. Also, by the above, the representation on $V_{\alpha}$ must have 0 as a weight, hence it is odd dimensional as asserted in the first case.

2. $K_{0} / H_{1}=(T \cdot S U(m+1)) /(T \cdot U(m))=\mathbb{C P}^{m}, m \geq 1, L=S U(m+1)$.

If $N \subset T \cdot S U(m+1)$ acts transitively on $\mathbb{C P}^{m}$, then $S^{1} \cdot N \subset U(m+1)$ acts transitively 
on $S^{2 m+1} \subset \mathbb{C}^{m+1}$ and hence must be one of the entries in Table 1 . Since $L \not \subset N$, we must have $N=T^{\prime} \cdot S p(k) \subset T \cdot S U(2 k)$ where $2 k=m+1$ and $T^{\prime} \subset T$ is at most one dimensional. That is, $N \cap L=S p(k)$, and $k \geq 2$ because $L \not \subset N$.

Let $\mathfrak{t} \subset \mathfrak{s u}(2 k)$ be the Lie algebra of the maximal torus consisting of all diagonal matrices. Note that any element $0 \neq X_{\mathfrak{m}} \in \mathfrak{m}_{1} \subset \mathfrak{s u}(2 k)$ is conjugate to a multiple of $X_{0}:=\operatorname{diag}(i,-i, 0, \ldots, 0) \in \mathfrak{t}$. Decompose

$$
V_{\alpha}=\bigoplus_{\lambda} W_{\lambda}
$$

into the weight spaces w.r.t. the maximal torus $\mathfrak{t}$.

Since $k \geq 2$, any non-trivial representation $V_{\alpha}$ of $L=S U(2 k)$ must have a weight $\lambda_{0} \neq 0$ which is annihilated by $X_{0} \in \mathfrak{t}$ from above, so by our assumption, the stabilizer of each non-zero element of $W_{\lambda_{0}}$ must be conjugate to $N \cap L=S p(k)$ and hence has rank $k$. On the other hand, $W_{\lambda_{0}}$ is stabilized by a hyperplane of the maximal torus of $L=S U(2 k)$ and hence this stabilizer has rank at least $2 k-2$. It follows that $k=2$, and we may assume that the Lie algebra of the maximal torus of $\mathfrak{s p}(2) \subset \mathfrak{s u}(4)$ is spanned by $X_{0}=\operatorname{diag}(i,-i, 0,0)$ and $\operatorname{diag}(0,0, i,-i)$. That is, any weight $\lambda$ of $V_{\alpha}$ which is annihilated by $X_{0}=\operatorname{diag}(i,-i, 0,0) \in \mathfrak{t} \subset \mathfrak{s u}(4)$ must also be annihilated by $\operatorname{diag}(0,0, i,-i)$. From here it easily follows that the only irreducible representation of $L=S U(4)$ with this property is the 6 -dimensional one which is the second case.

3. $K_{0} / H_{1}=(T \cdot S p(m+1)) /(T \cdot S p(1) \cdot S p(m))=\mathbb{H P}^{m}, m \geq 1, L=S p(m+1)$.

If $N \subset T \cdot S p(m+1)$ acts transitively on $\mathbb{H P}^{m}$, then $S p(1) \cdot N \subset S p(1) \cdot S p(m+1)$ acts transitively on $S^{4 m+3} \subset \mathbb{H}^{m+1}$ and hence must be one of the entries in Table 1. From there is follows that $N=T^{\prime} \cdot S p(m+1)$ where $T^{\prime} \subset T$, so that $L \subset N$ which is impossible.

4. $K_{0} / H_{1}=G_{2} / S U(3)=S^{6}, L=G_{2}$.

Table 1 reveals that there is no proper subgroup of $G_{2}$ acting transitively on $S^{6}$, so that we must have $N=G_{2}=L$, which is again impossible.

5. $K_{0} / H_{1}=\operatorname{Spin}(7) / G_{2}=S^{7}, L=\operatorname{Spin}(7)$.

The only subgroups of $\operatorname{Spin}(7)$ which act transitively on $S^{7}$ are $S U(4) \cong \operatorname{Spin}(6)$, $S^{1} \cdot \operatorname{Sp}(2) \cong \operatorname{Spin}(2) \cdot \operatorname{Spin}(5)$ and $\operatorname{Sp}(2) \cong \operatorname{Spin}(5)$.

Let $\mathfrak{t}^{3} \subset \mathfrak{s p i n}(7)$ be the Lie algebra of a maximal torus $T^{3} \subset \operatorname{Spin}(7)$, and suppose w.l.o.g. that $X_{\mathfrak{m}} \in \mathfrak{t}^{3}$. If the representation of $\operatorname{Spin}(7)$ on $V_{\alpha}$ was of spin type, then all elements $0 \neq X_{\mathfrak{m}}$ would act by isomorphisms on $V_{\alpha}$, contradicting our assumption on $X_{\mathfrak{m}}$. Thus, $V_{\alpha}$ is not of spin type and hence has 0 as a weight and we let $V_{0} \subset$ $\mathfrak{s}_{1}=\bigoplus_{\alpha \in \Phi_{1}} V_{\alpha}$ be the space stabilized by $T^{3}$. Thus, the stabilizer $N$ of any element in $V_{0}$ must contain $T^{3}$, hence must be either $\operatorname{Spin}(6)$ or $\operatorname{Spin}(2) \cdot \operatorname{Spin}(5)$. Since none of these two groups is conjugate to a subgroup of the other, we can argue as in 
the first case to conclude that all $0 \neq Y \in V_{0}$ have the same subgroup as stabilizer, and this subgroup must be invariant under conjugation by the Weyl group $W$. But $\operatorname{Spin}(2) \cdot \operatorname{Spin}(5) \subset \operatorname{Spin}(7)$ is not invariant under $W$, hence $N=\operatorname{Spin}(6) \subset \operatorname{Spin}(7)$.

It follows that no long root of $\mathfrak{s o}(7)$ can be a weight of $V_{\alpha}$, and the only irreducible representation of $\operatorname{Spin}(7)$ which has 0 but no long root as a weight is the standard one of $S O(7)=\operatorname{Spin}(7) / \mathbb{Z}_{2}$ on $\mathbb{R}^{7}$ which is the third case.

6. $K_{0} / H_{1}=\operatorname{Spin}(9) / \operatorname{Spin}(8) \cong S O(9) / S O(8)=S^{8}, L=\operatorname{Spin}(9)$.

By Table $1, S O(9)$ and $\operatorname{Spin}(9)$ are the only groups acting transitively on $S^{8}$, hence we must have $N=\operatorname{Spin}(9)=L$ which cannot be the case.

Proposition 5.3 Let $H \subset K \subset G$ and $L \subset K_{0}$ be as in Proposition 5.2, and consider the decomposition of $\mathfrak{g}$ from (14). Suppose that $\alpha \in \Phi_{2}$. Then one of the following cases must hold.

1. $K_{0}=L=\operatorname{Spin}(n+1)$ acting on $S^{n}$ for some $n \geq 2$, and $V_{\alpha}$ is an irreducible representation of spin type, i.e., is not the lift of a representation of $S O(n+1)=\operatorname{Spin}(n+1) / \mathbb{Z}_{2}$.

2. $K_{0}=T \cdot S U(2)$ acting on $S^{3}, L=S U(2)=S p i n(3)$, and $V_{\alpha}$ is an irreducible representation of $L$ of spin type.

3. $K_{0}=T \cdot S p(2)$ acting on $S^{7}, L=S p(2)=\operatorname{Spin}(5)$, and $V_{\alpha}$ is an irreducible representation of $L$ of spin type.

4. $K_{0}=L=\operatorname{Spin}(7)$ acting on $S^{7}$, and $V_{\alpha}$ is an irreducible representation of $L$ of spin type.

5. $K_{0}=L=\operatorname{Spin}(9)$ acting on $S^{15}$, and $V_{\alpha}$ is an irreducible representation of $L$ of spin type.

In all cases, $L=\operatorname{Spin}(n)$ for some $n$, and $\mathbb{Z}_{2} \subset Z(\operatorname{Spin}(n))$ acts as $\pm I d$ on $V_{\alpha}$, where $\mathbb{Z}_{2}$ is the kernel of the covering $\operatorname{Spin}(n) \rightarrow S O(n)$.

Proof. Again, we go through the various possibilities for $K_{0}$ and $L$ from Table 1 . First note that the representation of $V_{\alpha}$ cannot have 0 as a weight, since all elements $0 \neq X_{\mathfrak{m}} \in \mathfrak{m}_{1}$ are conjugate to elements of the maximal torus which would annihilate the 0 -weight space.

1. $L=K_{0}=S O(n+1)$ or $\operatorname{Spin}(n+1), n \geq 2$.

Any element $X_{\mathfrak{m}} \in \mathfrak{m}_{1}=\mathfrak{m}$ is conjugate to an element of the maximal Lie algebra of $\mathfrak{s o}(n+1)$ corresponding to the weight element $\theta_{1}$. Every irreducible representation of $\operatorname{Spin}(n+1)$ which is not of spin type and does not have 0 as a weight has all $\theta_{i}$ with $i=1, \ldots,[(n+1) / 2]$ as weights.

If $n \geq 3$, then $\theta_{2}$ is a weight which is annihilated by $X_{\mathfrak{m}}$, contradicting our assumption. If $n=2$, then any representation of $\operatorname{Spin}(3) \cong S U(2)$ which does not have 0 as a weight is of spin type. 
2. $K_{0}=T \cdot S U(m+1), m \geq 1, L=S U(m+1)$.

Any element $X_{\mathfrak{m}} \in \mathfrak{m}_{1}$ is conjugate to a multiple of $\operatorname{diag}(i,-i, 0, \ldots, 0) \in \mathfrak{t}$ which is the element of the maximal Lie algebra of $\mathfrak{s u}(m+1)$ corresponding to the weight element $\theta_{1}-\theta_{2}$. Every irreducible representation of $S U(m+1)$ has a weight of the form $\lambda=\theta_{1}+\ldots+\theta_{k}$ for some $k \leq m$. If $k \geq 2$, then $\left\langle\theta_{1}-\theta_{2}, \lambda\right\rangle=0$ so that $X$ acts trivially on the weight space $W_{\lambda}$ which is impossible. Thus, we must have $k=1$.

In this case, all elements $\theta_{i}, i=1, \ldots, m+1$ are weights, and if $m \geq 2$, then $\lambda=\theta_{3}$ is a weight with $\left\langle\theta_{1}-\theta_{2}, \lambda\right\rangle=0$, so that $X_{\mathfrak{m}}$ acts trivially on $W_{\lambda}$ which is impossible.

Thus, we must have $m=1$, i.e., $L=S U(2)=\operatorname{Spin}(3)$, and since 0 is not a weight of $V_{\alpha}$, it follows that $V_{\alpha}$ is even dimensional and therefore of spin type.

3. $K_{0}=T \cdot S p(m+1), m \geq 1, L=S p(m+1)$.

Any element $X_{\mathfrak{m}} \in \mathfrak{m}_{1}$ is conjugate to a multiple of $\operatorname{diag}(i, i, 0, \ldots, 0) \in \mathfrak{t}$ which is the element of the maximal torus of $\mathfrak{s p}(m+1)$ corresponding to the weight element $\theta_{1}+\theta_{2}$. Every irreducible representation of $S p(m+1)$ which does not have 0 as a weight has $\theta_{i}, i=1, \ldots, m+1$ as weights. Thus, if $m \geq 2$, then $\lambda=\theta_{3}$ is a weight, and since $\left\langle\theta_{1}+\theta_{2}, \lambda\right\rangle=0$, it follows that $X$ acts trivially on the weight space $W_{\lambda}$ which is impossible. Thus, we must have $m=1$, hence $L=\operatorname{Sp}(2)=\operatorname{Spin}(5)$.

Now it is easy to see that a representation of $\operatorname{Spin}(5)$ factors through $S O(5)$ if and only if it has 0 as a weight. Since we assume this not to be the case, $V_{\alpha}$ must be a spin type representation of $L=\operatorname{Sp}(2)=\operatorname{Spin}(5)$.

4. $K_{0}=L=G_{2}$.

Every representation of $G_{2}$ has 0 as a weight, so this case is impossible.

5. $K_{0}=L=\operatorname{Spin}(7)$ or $K_{0}=L=\operatorname{Spin}(9)$.

An irreducible representation of $\operatorname{Spin}(2 k+1)$ factors through a representation of $S O(2 k+1)=\operatorname{Spin}(2 k+1) / \mathbb{Z}_{2}$ if and only if it has 0 as a weight. Since $V_{\alpha}$ does not have 0 as a weight, we conclude that it must be a representation of spin type.

For the final assertion, note that $\mathbb{Z}_{2}=\operatorname{ker}(\operatorname{Spin}(n) \rightarrow S O(n))$ acts non-trivially in all cases, and since $\mathbb{Z}_{2} \subset Z(\operatorname{Spin}(n))$, it follows from Schur's Lemma that it must act on $V_{\alpha}$ as a multiple of the identity.

Corollary 5.4 Let $H \subset K \subset G$ and $L \subset K_{0} \subset K$ be as in Proposition 5.2, and consider the decomposition of $\mathfrak{g}$ from (14). Then there is an element $\sigma \in L$ such that $\sigma^{2}=1$, and such that $\left.A d_{\sigma}\right|_{\mathfrak{n}(\mathfrak{l}) \oplus \mathfrak{s}_{1}}=I d$ and $\left.A d_{\sigma}\right|_{\mathfrak{s}_{2}}=-I d$.

In particular, $\left(\mathfrak{g}, \mathfrak{n}(\mathfrak{l}) \oplus \mathfrak{s}_{1}\right)=:\left(\mathfrak{g}, \mathfrak{n}_{0}\right)$ is a symmetric pair whose reflection is given by $A d_{\sigma}$. 
Proof. If $L \neq \operatorname{Spin}(n)$ for some $n$, then $\mathfrak{s}_{2}=0$ by Proposition 5.3, so that in this case the claim holds for $\sigma=1 \in L$.

If $L \cong \operatorname{Spin}(n)$ for some $n$, then let $\sigma \in L$ be the non-trivial element in the kernel of the covering $\operatorname{Spin}(n) \rightarrow S O(n)$. By Proposition [5.3, $A d_{\sigma}$ acts as $-I d$ on $\mathfrak{s}_{2}$.

Also, $A d_{\sigma}$ acts trivially on the normalizer $\mathfrak{n}(\mathfrak{l})$ since $\sigma \in Z(L)$. Finally, if $\mathfrak{s}_{1} \neq 0$, then by Proposition 5.2 we have in all cases that $\mathfrak{s}_{1}$ is a representation of $S O(n)=\operatorname{Spin}(n) / \mathbb{Z}_{2}$, so that $\left.A d_{\sigma}\right|_{\mathfrak{s}_{1}}=I d$.

It thus follows that $\mathfrak{n}_{0}:=\mathfrak{n}(\mathfrak{l}) \oplus \mathfrak{s}_{1} \subset \mathfrak{g}$ is a Lie subalgebra, and we let $N_{0} \subset G$ be the corresponding connected Lie subgroup, so that $G / N_{0}$ is a symmetric space whose dimension equals that of $\mathfrak{s}_{2}$. Let us consider the various possibilities for $N_{0}$ is more detail.

Proposition 5.5 Let $H \subset K \subset G$ and $L \subset K_{0} \subset K$ be as in Proposition 5.2, and let $N_{0} \subset G$ be the connected subgroup with Lie algebra $\mathfrak{n}_{0}=\mathfrak{n}(\mathfrak{l}) \oplus \mathfrak{s}_{1}$. Then there are normal subgroups $\tilde{L}, \tilde{N}_{0} \subset N_{0}$ such that $N_{0}=\tilde{L} \cdot \tilde{N}_{0}$ and $L \subset \tilde{L}$, and one of the following holds.

1. $\tilde{L}=L$,

2. $K_{0} / H_{0}=S U(2) \cdot S U(2)^{\prime} / \triangle S U(2)=S^{3}, L=K_{0}$, and $\tilde{L}=\hat{L} \cdot S U(2)^{\prime}$ for some normal subgroup $\hat{L} \subset \tilde{L}$, where $S U(2) \subset \hat{L}$ is such that $Z(S U(2)) \subset Z(\hat{L})$.

3. $K_{0} / H_{0}=(T \cdot S U(4)) /(T \cdot S U(3)), \operatorname{dim} T \leq 1, L=S U(4) \cong \operatorname{Spin}(6)$ and $\tilde{L}=\operatorname{Spin}(7)$, where $L=\operatorname{Spin}(6) \subset \operatorname{Spin}(7)$ by the standard inclusion. Moreover, the adjoint action of $\tilde{L}$ on $\mathfrak{s}_{2}$ is of spin type.

4. $K_{0} / H_{0}=\operatorname{Spin}(7) / G_{2}, L=K_{0}=\operatorname{Spin}(7)$ and $\tilde{L} \in\{\operatorname{SO}(8), \operatorname{Spin}(8), \operatorname{Spin}(9)\}$. Moreover, for each $\tilde{L}$-irreducible subspace $\tilde{V} \subset \mathfrak{s}_{2}$, one of the following holds:

(a) $\operatorname{Spin}(7) \hookrightarrow \operatorname{Spin}(7+p)$ is the lift of the standard inclusion $S O(7) \subset S O(7+p)$ for $p=1,2$, and the action of $\tilde{L}$ on $\tilde{V}$ is of spin type, or

(b) $\operatorname{Spin}(7) \subset S O(8)=\tilde{L}(\operatorname{Spin}(7) \subset \operatorname{Spin}(8)=\tilde{L}$, respectively) is given by (the lift of) the spin representation of $\operatorname{Spin}(7)$, and the representation of $\tilde{L}$ on $\tilde{V}$ is (the lift of) a representation of $S O(8) \supset S p i n(7)$ which does not have 0 as a weight.

Proof. If $\mathfrak{s}_{1}=0$, then $\mathfrak{n}_{0}=\mathfrak{n}(\mathfrak{l})$, hence $\mathfrak{l} \triangleleft \mathfrak{n}_{0}$, so that the first case holds. If $\mathfrak{s}_{1} \neq 0$, then according to Proposition 5.2 we have only few possibilities which we shall investigate now.

1. $L=S U(2) \cdot S U(2)^{\prime}$, and $\left[\mathfrak{s u}(2)^{\prime}, \mathfrak{s}_{1}\right]=0$ so that $\mathfrak{s u}(2)^{\prime} \triangleleft \mathfrak{n}_{0}$ and hence, $N_{0}:=\hat{L} \cdot S U(2)^{\prime}$. $\tilde{N}_{0}$ where $\hat{L} \subset N_{0}$ is the normal subgroup generated by $S U(2)$. Since $Z(S U(2))$ acts trivially on all irreducible $V_{\alpha} \subset \mathfrak{s}_{1}$ as $V_{\alpha}$ is odd dimensional, $Z(S U(2)) \subset Z(\hat{L})$ follows.

2. If $L=S U(4)$, then $V_{\alpha} \cong \mathbb{R}^{6}$ with the standard representation of $S O(6)=S U(4) / \mathbb{Z}_{2}$. Since there is no $L$-equivariant map $\mathbb{R}^{6} \otimes \mathbb{R}^{6} \rightarrow \mathbb{R}^{6}$, it follows that $\left(\mathfrak{n}_{0}, \mathfrak{n}(\mathfrak{l})\right)$ is a symmetric pair. 
Therefore, we have a decomposition $\mathfrak{n}_{0}=\mathfrak{g}_{1} \oplus \ldots \oplus \mathfrak{g}_{k}$ such that $\mathfrak{n}(\mathfrak{l})=\left(\mathfrak{n}(\mathfrak{l}) \cap \mathfrak{g}_{1}\right) \oplus$ $\ldots \oplus\left(\mathfrak{n}(\mathfrak{l}) \cap \mathfrak{g}_{k}\right)$, and such that $\left(\mathfrak{g}_{i}, \mathfrak{g}_{i} \cap \mathfrak{n}(\mathfrak{l})\right)$ is an irreducible symmetric pair. Since $\mathfrak{l} \triangleleft \mathfrak{n}(\mathfrak{l})$ is simple, it follows that it must be contained in one of these summands, say, $\mathfrak{l} \subset \mathfrak{n}(\mathfrak{l}) \cap \mathfrak{g}_{1}$. Therefore, if we let $\tilde{\mathfrak{l}}:=\mathfrak{g}_{1}$ and $\tilde{\mathfrak{n}}_{0}:=\mathfrak{g}_{2} \oplus \ldots \oplus \mathfrak{g}_{k}$ and $\tilde{L}, \tilde{N}_{0} \subset N_{0}$ be the corresponding normal subgroups, then $N_{0}=\tilde{L} \cdot \tilde{N}_{0}$.

Consider now the irreducible symmetric pair $(\tilde{\mathfrak{l}}, \mathfrak{n}(\mathfrak{l}) \cap \tilde{\mathfrak{l}})$. The isotropy group contains $\mathfrak{l}=\mathfrak{s o}(6)=\mathfrak{s u}(4)$ as an ideal, whose isotropy representation is given by direct sums of the 6 -dimensional representation. From the classification of irreducible symmetric spaces $([\underline{\mathrm{H}}])$ it follows that $\tilde{\mathfrak{l}}=\mathfrak{s o}(6+p)$ and $\mathfrak{n}(\mathfrak{l}) \cap \tilde{\mathfrak{l}}=\mathfrak{s o}(6) \oplus \mathfrak{s o}(p)$ for some $p \geq 1$.

Note that $\mathfrak{h}_{1}=\mathfrak{u}(3) \subset \mathfrak{s u}(4) \cong \mathfrak{s o}(6)$ with the standard embedding, and pick a basis of $\mathbb{R}^{6}$ such that $\mathfrak{z}(\mathfrak{u}(3))=\mathbb{R}\left(E_{12}+E_{34}+E_{56}\right)$ where as before, $E_{r s}$ denotes the skewsymmetric matrix of rank two with $E_{r s} e_{r}=e_{s}$ and $E_{r s} e_{s}=-e_{r}$, where $\left(e_{r}\right)$ is the standard basis. If $p \geq 2$ so that $6+p \geq 8$, then we let

$$
X_{\mathfrak{m}}:=E_{13}-E_{24}, Y_{\mathfrak{m}}:=E_{14}+E_{23}, X_{\mathfrak{s}}:=\sqrt{2}\left(E_{17}+E_{38}\right), Y_{\mathfrak{s}}:=-\sqrt{2}\left(E_{27}+E_{48}\right) .
$$

One easily verifies that $X_{\mathfrak{m}}, Y_{\mathfrak{m}} \in \mathfrak{s o}(6) \cap \mathfrak{u}(3)^{\perp}=\mathfrak{m}_{1}, X_{\mathfrak{s}}, Y_{\mathfrak{s}} \in \mathfrak{s}$ and that for $X:=$ $X_{\mathfrak{m}}+X_{\mathfrak{s}}$ and $Y:=Y_{\mathfrak{m}}+Y_{\mathfrak{s}}$ we have $[X, Y]=0$, which is impossible according to (4). Thus, we must have $p=1$ and hence $\tilde{\mathfrak{l}} \cong \mathfrak{s o}(7)$. Since the corresponding group $\tilde{L}$ must contain $L=\operatorname{Spin}(6)$ as a subgroup, we cannot have $\tilde{L} \cong S O(7)$, so that $\tilde{L} \cong \operatorname{Spin}(7)$ as claimed.

The representation of $\tilde{L}=\operatorname{Spin}(7)$ on $\mathfrak{s}_{2}$ must be of spin type. For if this was not the case, then this representation would have 0 as a weight, hence so would its restriction to $L=\operatorname{Spin}(6)$. However, $L$ acts on $\mathfrak{s}_{2}$ of spin type which is a contradiction.

3. If $L=\operatorname{Spin}(7)$, then $V_{\alpha} \cong \mathbb{R}^{7}$ with the standard representation of $S O(7)=\operatorname{Spin}(7) / \mathbb{Z}_{2}$. Arguing as in the previous case, we conclude that there is a normal subgroup $\tilde{L} \subset N_{0}$ with Lie algebra $\tilde{\mathfrak{l}}=\mathfrak{s o}(7+p)$ for some $p \geq 1$, and the inclusion $L \hookrightarrow \tilde{L}$ is the lift of the standard inclusion $S O(7) \subset S O(7+p)$. Thus, it follows from Proposition 4.1 that $p \leq 2$.

If $p=2$ then $\tilde{\mathfrak{l}}=\mathfrak{s o}(9)$ hence $\tilde{L}=\operatorname{Spin}(9)$ and in the notation of section 4 , $L=$ $\operatorname{Spin}_{0}(7) \subset \operatorname{Spin}(9)$. Since $Z(\operatorname{Spin}(9))=Z\left(\operatorname{Spin}_{0}(7)\right)=\mathbb{Z}_{2}$, the restriction of a representation of $\operatorname{Spin}(9)$ to $\operatorname{Spin}_{0}(7)$ is of spin type if and only if the representation of $\operatorname{Spin}(9)$ itself is of spin type showing the claim in this case.

If $p=1$ then $\tilde{L}$ is the quotient of $\operatorname{Spin}(8)$ by a subgroup of $Z(\operatorname{Spin}(8))=\mathbb{Z}_{2} \oplus \mathbb{Z}_{2}$. Let $\tilde{V} \subset \mathfrak{s}_{2}$ be a $\tilde{L}$-irreducible subspace, and let $\Gamma \subset Z(\operatorname{Spin}(8))$ be the kernel of the representation of $\tilde{L}$ on $\tilde{V}$. Since the restriction of this representation to $\operatorname{Spin}(7)$ is of spin type, it follows that $\Gamma \cap Z(\operatorname{Spin}(7))=0$. Therefore, $\Gamma \subsetneq Z(\operatorname{Spin}(8))$, so that this representation cannot have 0 as a weight. If $\Gamma=0$, then the representation of $\tilde{L}=\operatorname{Spin}(8)$ on $\mathfrak{s}_{2}$ is of spin type. If $\Gamma \neq 0$, then we must have $\Gamma \cong \mathbb{Z}_{2}$, and this action is given by a representation of $S O(8)=\operatorname{Spin}(8) / \Gamma$ which does not have 0 as a weight. Moreover, the inclusion $\operatorname{Spin}(7) \hookrightarrow S O(8)=\operatorname{Spin}(8) / \Gamma$ is the spin representation. 
Table 2

LiST OF IRREDUCIBLE SYMMETRIC SPACES $G_{1} /\left(\operatorname{Spin}(n) \cdot N^{\prime}\right)$ WITH SPIN TYPE ISOTROPY

\begin{tabular}{|c|c|c|c|}
\hline & $L \cong \operatorname{Spin}(n)$ & $G_{1} /\left(\operatorname{Spin}(n) \cdot N^{\prime}\right)$ & isotropy representation of $L$ \\
\hline 1 & $S U(2) \cong \operatorname{Spin}(3)$ & $S U(p+2) / S(U(2) \cdot U(p)), p \geq 1$ & $\mathbb{C}^{2}$ (standard repr.) \\
\hline 2 & $S U(2) \cong \operatorname{Spin}(3)$ & $\begin{array}{c}S O(p+4) /(S O(4) \cdot S O(p)), p \geq 1 \\
S U(2) \hookrightarrow S O(4) \times 1\end{array}$ & $\mathbb{C}^{2}$ (standard repr.) \\
\hline 3 & $\operatorname{Sp}(1) \cong \operatorname{Spin}(3)$ & $S p(p+1) /(S p(1) \cdot S p(p)), p \geq 1$ & $\mathbb{C}^{2}$ (standard repr.) \\
\hline 4 & $S U(2) \cong \operatorname{Spin}(3)$ & $\begin{array}{c}G_{2} / S O(4) \\
L=S U(2)_{1} \subset S O(4)\end{array}$ & $\mathbb{C}^{2}$ (standard repr.) \\
\hline 5 & $S U(2) \cong \operatorname{Spin}(3)$ & $\begin{array}{c}G_{2} / S O(4) \\
L=S U(2)_{3} \subset S O(4)\end{array}$ & $\mathbb{C}^{4} \cong \odot^{3}\left(\mathbb{C}^{2}\right)$ \\
\hline 6 & $S U(2) \cong \operatorname{Spin}(3)$ & $F_{4} /(S U(2) \cdot S p(3))$ & $\mathbb{C}^{2}$ (standard repr.) \\
\hline 7 & $S U(2) \cong \operatorname{Spin}(3)$ & $E_{6} /(S U(2) \cdot S U(6))$ & $\mathbb{C}^{2}$ (standard repr.) \\
\hline 8 & $S U(2) \cong \operatorname{Spin}(3)$ & $E_{7} /(S U(2) \cdot \operatorname{Spin}(12))$ & $\mathbb{C}^{2}$ (standard repr.) \\
\hline 9 & $S U(2) \cong \operatorname{Spin}(3)$ & $E_{8} /\left(S U(2) \cdot E_{7}\right)$ & $\mathbb{C}^{2}$ (standard repr.) \\
\hline 10 & $S p(2) \cong \operatorname{Spin}(5)$ & $S p(p+2) /(S p(2) \cdot S p(p)), p \geq 1$ & $\mathbb{H}^{2}$ (standard repr.) \\
\hline 11 & $S U(4) \cong \operatorname{Spin}(6)$ & $S U(p+4) / S(U(4) \cdot U(p)), p \geq 1$ & $\Delta_{6} \cong \mathbb{C}^{4}$ (spin repr. $)$ \\
\hline 12 & $\operatorname{Spin}(8)$ & $\operatorname{Spin}(p+9) /(\operatorname{Spin}(8) \cdot \operatorname{Spin}(p+1)), p \geq 0$ & $\Delta_{8} \cong \mathbb{R}^{8}$ (spin repr.) \\
\hline 13 & $\operatorname{Spin}(9)$ & $F_{4} / \operatorname{Spin}(9)$ & $\Delta_{9}($ spin repr.) \\
\hline 14 & $\operatorname{Spin}(10)$ & $E_{6} /(\operatorname{Spin}(10) \cdot U(1))$ & $\Delta_{10}^{+}($spin repr. $)$ \\
\hline 15 & $\operatorname{Spin}(12)$ & $E_{7} /(\operatorname{Spin}(12) \cdot S U(2))$ & $\Delta_{12}^{+}($spin repr. $)$ \\
\hline 16 & $\operatorname{Spin}(16)$ & $E_{8} / \operatorname{Spin}(16)$ & $\Delta_{16}^{+}$(spin repr.) \\
\hline
\end{tabular}

Proposition 5.6 Let $H \subset K \subset G$ and $L \subset K_{0} \subset K$ be as in Proposition 5.2. As before, let $N_{0} \subset G$ be the connected subgroup with Lie algbera $\mathfrak{n}(\mathfrak{l}) \oplus \mathfrak{s}_{1}$ and let $\tilde{L} \subset N_{0}$ be the normal subgroup from Proposition 5.5. Furthermore, suppose that $L$ is not isomorphic to $S U(2) \cdot S U(2)$.

Then either $\tilde{L} \subset G$ is a normal subgroup, or $L=\tilde{L}$ and there is a normal subgroup $G_{1} \subset G$ such that $L \subset N_{0} \cap G_{1}$ is normal, and $G_{1} /\left(N_{0} \cap G_{1}\right)$ is one of the entries of Table 2.

Proof. If $\mathfrak{s}_{2}=0$ then $G=N_{0}$ and hence, $\tilde{L} \subset N_{0}=G$ is a normal subgroup by Proposition 5.5 ,

Let us suppose that $\mathfrak{s}_{2} \neq 0$ and $\tilde{L}=L$. By Proposition 5.3 , it follows that $L=\operatorname{Spin}(n)$ for some $n$, and $L$ acts on $\mathfrak{s}_{2}$ of spin type. Since we excluded the case $n=4, L=\tilde{L}$ is simple. 
Thus, if we decompose the symmetric space $G / N_{0}$ from Corollary 5.4 as

$$
G=G_{1} \cdot \ldots \cdot G_{k}, N_{0}:=N_{1} \cdot \ldots \cdot N_{k}, \text { with } N_{i}=G_{i} \cap N
$$

such that $G_{i} / N_{i}$ are irreducible symmetric spaces, then we may assume w.l.o.g. that $L \subset N_{1}$ is normal, so that $N_{1}=L \cdot N^{\prime}$ for some normal subgroup $N^{\prime} \subset N_{1}$. That is, $G_{1} / N_{1}=$ $G_{1} /\left(\operatorname{Spin}(n) \cdot N^{\prime}\right)$ is an irreducible symmetric space such that the restriction of the isotropy representation to $\operatorname{Spin}(n)$ is of spin type. From the classification of irreducible symmetric spaces $([\mathrm{H}])$, we conclude that $G_{1} / N_{1}$ must be an entry of Table 2.

It remains to exclude the case $\mathfrak{s}_{2} \neq 0$ and $L \subsetneq \tilde{L}$. By Proposition 5.5, it follows again that $\tilde{L}$ is simple as we excluded the case $L=S U(2) \cdot S U(2)$, so that as before we may assume that there is a normal subgroup $G_{1} \subset G$ such that $G_{1} / N_{1}$ is an irreducible symmetric space, where $N_{1}=\tilde{L} \cdot N^{\prime}$. In particular, it follows that all $\tilde{L}$-irreducible subspaces of $\tilde{V} \subset \mathfrak{s}_{2}$ are equivalent.

Let us work through the possibilities for $\tilde{L}$ given in Proposition 5.5. If $\tilde{L}=\operatorname{Spin}(n)$ with $n \in\{7,8,9\}$ acts on $\mathfrak{s}_{2}$ of spin type, then $G_{1} / N_{1}=G_{1} /\left(\operatorname{Spin}(n) \cdot N^{\prime}\right)$ must be an entry of Table 2, whence the only possibility is $n=9$ and $G_{1}=F_{4}$. However, the triple $\left(H_{0} \subset K_{0} \subset G\right)=\left(G_{2} \subset \operatorname{Spin}_{0}(7) \subset F_{4}\right)$ was shown not to satisfy (4) in Proposition 4.1, so that this case is impossible.

Finally, if $\tilde{L} \in\{S O(8), \operatorname{Spin}(8)\}$ acts on $\mathfrak{s}_{2}$ as (the lift of) a representation of $S O(8)$ which does not have 0 as a weight, then again, the classification of irreducible symmetric spaces implies that $G_{1} / N_{1}=S O(p+9) /(S O(8) \cdot S O(p+1))$ for some $p \geq 0$, corresponding to the triple $\left(H_{0} \subset K_{0} \subset G\right)=\left(G_{2} \subset \operatorname{Spin}_{ \pm}(7) \subset S O(p+9)\right)$ which was also shown not to satisfy (4) in Proposition 4.1, so the proof is completed.

Proposition 5.7 Let $H \subset K \subset G$ and $L \subset K_{0} \subset K$, and $N_{0} \subset G$ be as in Proposition 5.6 and assume that $L=\tilde{L}$. Furthermore, let $G_{1} /\left(N_{0} \cap G_{1}\right)$ be the irreducible symmetric space from that proposition. Then one of the following cases holds.

1. $L=S p(1) \subset S p(p+1)$ and $G_{1} /\left(N_{0} \cap G_{1}\right)=S p(p+1) /(S p(1) \cdot S p(p))$ for some $p \geq 1$,

2. $L=S U(2)_{3} \subset G_{2}$ and $G_{1} /\left(N_{0} \cap G_{1}\right)=G_{2} / S O(4)$,

3. $L=\operatorname{Spin}(6) \cong S U(4) \subset S U(5)$, and $G_{1} /\left(N_{0} \cap G_{1}\right)=S U(5) / U(4)$,

4. $L=\operatorname{Spin}(8) \subset \operatorname{Spin}(p+9)$ for $p \in\{0,1,2\}$, and $G_{1} /\left(N_{0} \cap G_{1}\right)=\operatorname{Spin}(p+9) /(\operatorname{Spin}(8)$. $\operatorname{Spin}(p+1))$, and the action of $K_{0}=L$ on $\mathbb{R}^{8}$ is the spinor representation.

That is, of Table 2 only the entries 3, 5, 11 for $p=1$ and 12 for $p \in\{0,1,2\}$ can occur.

Proof. In order to show the proposition, we have to exclude all but these possibilities in Table 2. 


\section{Entries 1, 2, 4, 6, 7, 8, 9 of Table 2}

In this case, $G_{1}$ is a compact simple Lie group whose Lie algebra does not have type $C_{n}$ in the classification of Dynkin diagrams, and $L=S U(2)$ is the subgroup generated by a long root $\alpha$. By our assumption, there is a long $\operatorname{root} \beta$ with $\langle\alpha, \beta\rangle=1$, so that the root spaces of $\alpha$ and $\beta$ generate a subgroup of $G_{1}$ which is isomorphic to $S U(3)$ and contains $L=S U(2)$ as subgroup. Since the normalizer of $L \subset S U(3)$ is $U(2)$, it follows that $\mathfrak{s} \cap \mathfrak{s u}(3) \subset \mathfrak{s}$ containes the orthogonal complement of $\mathfrak{u}(2) \subset \mathfrak{s u}(3)$. If $\mathfrak{h}_{0} \subset \mathfrak{l}$ is non-trivial, then it is one-dimensional and w.l.o.g. is spanned by $\operatorname{diag}(i,-i, 0) \in$ $\mathfrak{s u}(2) \subset \mathfrak{s u}(3)$. Therefore, the elements

$$
X:=\left(\begin{array}{rr|r}
0 & 1 & 1 \\
-1 & 0 & 1 \\
\hline-1 & -1 & 0
\end{array}\right) \text { and } Y:=\left(\begin{array}{rr|r}
0 & i & -i \\
i & 0 & i \\
\hline-i & i & 0
\end{array}\right)
$$

are contained in $\mathfrak{m}_{1} \oplus \mathfrak{s}$ and satisfy $[X, Y]=0$, where

$$
X_{\mathfrak{m}}:=\left(\begin{array}{rr}
0 & 1 \\
-1 & 0
\end{array}\right) \text {, and } Y_{\mathfrak{m}}:=\left(\begin{array}{cc}
0 & i \\
i & 0
\end{array}\right)
$$

are independent elements of $\mathfrak{m}_{1} \subset \mathfrak{l}$, contradicting (4).

2. Entry 10 of Table 2: $G_{1}=S p(p+2), L=S p(2)$.

In this case, $L$ acts either on $S^{7}$ in which case $\mathfrak{h}_{0}=\mathfrak{s p}(1) \subset \mathfrak{s p}(2)$, or on $S^{4}$ in which case $\mathfrak{h}_{0}=\mathfrak{s p}(1) \oplus \mathfrak{s p}(1) \subset \mathfrak{s p}(2)$. In either case, for $p=1$ we can regard the matrices $X, Y$ from (15) as elements of $\mathfrak{m}_{1} \oplus \mathfrak{s} \subset \mathfrak{s p}(3)$ with $[X, Y]=0$ and $X_{\mathfrak{m}} \wedge Y_{\mathfrak{m}} \neq 0$, violating (4). Using the embedding $\mathfrak{s p}(3) \hookrightarrow \mathfrak{s p}(p+2)$ for $p>1$ we can rule out this case as well.

3. Entry 11 of Table 2: $G_{1}=S U(p+4), L=S U(4)$.

In this case, $L$ acts either on $S^{7}$ so that $H_{0}=S U(3)$, or it acts on $S^{5}$ so that $H_{0}=S p(2)$. In the first case, the triple $H_{0} \subset K_{0} \subset G$ is given as $S U(3) \subset S U(4) \subset S U(p+4)$. Then we can choose a subgroup $G^{\prime} \cong S U(3) \subset S U(p+4)$ such that $H_{0} \cap G^{\prime}=1$ and $K_{0} \cap G^{\prime}=S U(2)$. Then the elements $X, Y \in \mathfrak{s u}(3) \cong \mathfrak{g}^{\prime} \subset \mathfrak{g}$ from (15) show that (4) is violated.

In the second case, the triple $H_{0} \subset K_{0} \subset G_{1}$ is given by $S p(2) \subset S U(4) \subset S U(p+4)$. We assert that for $p \geq 2, G_{1}$ contains a subgroup $G^{\prime}=S U(3) \cdot S U(3)$ such that $K_{0} \cap G^{\prime}=S U(2) \cdot S U(2)$ and $H_{0} \cap G^{\prime}=\triangle S U(2)$. Once this is shown, we can pick the pairs $\hat{X}:=(X,-X), \hat{Y}:=(Y,-Y) \in \mathfrak{g}^{\prime}$ with $X, Y \in \mathfrak{s u}(3)$ from (15), so that $\hat{X}, \hat{Y} \in\left(\mathfrak{h}_{0} \oplus \mathfrak{z}\left(\mathfrak{h}_{0}\right)\right)^{\perp} \subset \mathfrak{m} \oplus \mathfrak{s}$, satisfy $[\hat{X}, \hat{Y}]=0$ and $\hat{X}_{\mathfrak{m}}=\left(X_{\mathfrak{m}},-X_{\mathfrak{m}}\right), \hat{Y}_{\mathfrak{m}}=\left(Y_{\mathfrak{m}},-Y_{\mathfrak{m}}\right)$ are linearly independent, contradicting (44).

To see the existence of $G^{\prime}$, choose a complex orthonormal basis $\left\{e_{i}, f_{i}, g_{r} \mid i=1,2, r=\right.$ $1, \ldots p\}$ of $\mathbb{C}^{p+4}$ such that $\mathfrak{s u}(4) \subset \mathfrak{s u}(p+4)$ is the stabilizer of $\operatorname{span}\left(g_{r}\right)$, and $\mathfrak{s p}(2) \subset$ $\mathfrak{s u}(4)$ is the stabilizer of the quaternionic structure $J: \operatorname{span}\left(e_{i}, f_{i}\right) \rightarrow \operatorname{span}\left(e_{i}, f_{i}\right)$ 
defined as the antilinear map with $J e_{i}=(-1)^{i+1} e_{i+1}$ and $J f_{i}=(-1)^{i+1} f_{i+1}$, taking indices mod 2. If we now define

$$
\mathfrak{g}^{\prime}:=\mathfrak{s u}(3) \oplus \mathfrak{s u}(3) \subset \operatorname{stab}\left(\operatorname{span}\left(e_{1}, f_{1}, g_{1}\right) \oplus \operatorname{span}\left(e_{2}, f_{2}, g_{2}\right)\right),
$$

then the properties $\mathfrak{s u}(4) \cap \mathfrak{g}^{\prime}=\mathfrak{s u}(2) \oplus \mathfrak{s u}(2)$ and $\mathfrak{s p}(2) \cap \mathfrak{g}^{\prime}=\triangle \mathfrak{s u}(2) \subset \mathfrak{s u}(2) \oplus \mathfrak{s u}(2)$ are easily verified.

4. Entry 12 of Table 2: $G_{1}=\operatorname{Spin}(p+8), L=\operatorname{Spin}(8)$.

In this case, the inclusion $H_{0} \subset K_{0} \subset G_{1}$ is given by $\operatorname{Spin}_{ \pm}(7) \subset \operatorname{Spin}(8) \subset \operatorname{Spin}(p+9)$, which violates (4) for $p \geq 3$ by Proposition 4.1.

5. Entry 13 of Table 2: $G_{1}=F_{4}, L=\operatorname{Spin}(9) \subset F_{4}$.

In this case, $\mathfrak{m}_{1}=\mathfrak{s o}(8)^{\perp} \cap \mathfrak{s o}(9)$. If there were no linearly independent $X, Y \in$ $\mathfrak{m}_{1} \oplus\left(\mathfrak{s} \cap \mathfrak{f}_{4}\right)$ with $[X, Y]=0$, then the normal homogeneous metric on $F_{4} / \operatorname{Spin}(8)$ would have positive curvature. However, by the classification of these spaces from [Be] this is not the case, so that we must have such elements.

Suppose that for all commuting elements $X, Y \in \mathfrak{m}_{1} \oplus\left(\mathfrak{s} \cap \mathfrak{f}_{4}\right)$ we have $X_{\mathfrak{m}} \wedge Y_{\mathfrak{m}}=0$ so that we may assume w.l.o.g. that $Y_{\mathfrak{m}}=0$. Then $0=[X, Y]=\left[X_{\mathfrak{m}}, Y_{\mathfrak{s}}\right]+\left[X_{\mathfrak{s}}, Y_{\mathfrak{s}}\right]$, and since $F_{4} / \operatorname{Spin}(9)$ is a symmetric space, so that $\left[X_{\mathfrak{s}}, Y_{\mathfrak{s}}\right] \in \mathfrak{s o}(9)$, whereas $\left[X_{\mathfrak{m}}, Y_{\mathfrak{s}}\right] \in$ $\mathfrak{s} \subset \mathfrak{s o}(9)^{\perp}$ this implies that $\left[X_{\mathfrak{m}}, Y_{\mathfrak{s}}\right]=\left[X_{\mathfrak{s}}, Y_{\mathfrak{s}}\right]=0$. But the non-zero elements of $\mathfrak{m}_{1}=\mathfrak{s o}(8)^{\perp} \subset \mathfrak{s o}(9)$ act on $\mathfrak{s}$ by isomorphisms, hence $\left[X_{\mathfrak{m}}, Y_{\mathfrak{s}}\right]=0$ and $Y_{\mathfrak{s}} \neq 0$ implies that $X_{\mathfrak{m}}=0$. Furthermore, $F_{4} / \operatorname{Spin}(9)$ is a symmetric space of rank one, hence $\left[X_{\mathfrak{s}}, Y_{\mathfrak{s}}\right]=0$ implies that $X_{\mathfrak{s}}, Y_{\mathfrak{s}}$ are linearly dependent which is impossible.

Therefore, we must have $[X, Y]$ and $X_{\mathfrak{m}} \wedge Y_{\mathfrak{m}} \neq 0$ which contradicts (4).

6. Entries 14, 15, 16 of Table 2: $G_{1}=E_{r}, L=K_{0}=\operatorname{Spin}(2 k), H_{0}=\operatorname{Spin}(2 k-1)$, where $(r, k) \in\{(6,5),(7,6),(8,8)\}$.

We assert that in all three cases, $G_{1}$ contains a subgroup $G^{\prime} \cong S U(3) \cdot S U(3)$ such that $S U(2) \cdot S U(2) \subset K_{0} \cap G^{\prime} \subset U(2) \cdot U(2)$, and $\triangle S U(2) \subset H_{0} \cap G^{\prime} \subset \triangle S U(2) \cdot$ $Z(U(2) \cdot U(2))$. This will be sufficient for our purposes: we choose $\hat{X}=(X,-X), \hat{Y}=$ $(Y,-Y) \in \mathfrak{m} \oplus \mathfrak{s}$ as in case 3 above to derive a contradiction to (4).

In order to see the existence of $G^{\prime} \subset G_{1}$, we fix the orthonormal basis $\theta_{1}, \ldots, \theta_{r}$ of the maximal torus of the Lie algebra $\mathfrak{e}_{r}$ such that

(i) the Lie algebra $\mathfrak{s o}(2 k) \subset \mathfrak{e}_{r}$ has weights $\pm \theta_{i} \pm \theta_{j}, 1 \leq i<j \leq k$,

(ii) the weights of the isotropy representation of $\operatorname{Spin}(2 k)$ are $\frac{1}{2}\left(\varepsilon_{1} \theta_{1}+\ldots+\varepsilon_{r} \theta_{k}\right)$, where $\varepsilon_{i}= \pm 1$ such that $\varepsilon_{1} \ldots \varepsilon_{k}=1$,

(iii) $\mathfrak{h}_{0}=\mathfrak{s o}(2 k-1) \subset \mathfrak{s o}(2 k)$ is the Lie algebra which stabilizes $\theta_{1}$.

Now we proceed by investigating the three cases separately. 
(a) Suppose $(r, k)=(6,5)$, corresponding to the Hermitean symmetric space $E_{6} / \operatorname{Spin}(10) \cdot U(1)$. Then $(\mathfrak{s o}(10) \oplus \mathfrak{u}(1))^{\perp}$ is a complex representation of $\mathfrak{s o}(10) \oplus$ $\mathfrak{u}(1)$, and the complex one-dimensional weight spaces $V_{\lambda} \subset(\mathfrak{s o}(10) \oplus \mathfrak{u}(1))^{\perp}$ for $\lambda=\frac{1}{2}\left(\varepsilon_{1} \theta_{1}+\ldots+\varepsilon_{r} \theta_{k}\right)$ are well defined. Moreover, $\left[V_{\lambda}, V_{\lambda^{\prime}}\right] \neq 0$ if and only if $\lambda-\lambda^{\prime}$ is a root of $\mathfrak{s o}(10)$; in this case, $\left[V_{\lambda}, V_{\lambda^{\prime}}\right]=\mathfrak{g}_{ \pm\left(\lambda-\lambda^{\prime}\right)} \oplus \mathfrak{t}^{1}$ for a one dimensional Lie algebra $\mathfrak{t}^{1} \subset \operatorname{span}\left(\lambda, \lambda^{\prime}\right) \oplus \mathfrak{u}(1)$, regarding $\lambda, \lambda^{\prime}$ as elements of the maximal torus of $\mathfrak{e}_{r}$. We define the weights

$$
\lambda_{1 / 2}:=\frac{1}{2}\left( \pm\left(\theta_{1}+\theta_{2}\right)+\theta_{3}+\theta_{4}+\theta_{5}\right), \text { and } \mu_{1 / 2}:=\frac{1}{2}\left( \pm\left(\theta_{1}-\theta_{2}\right)-\theta_{3}-\theta_{4}-\theta_{5}\right) .
$$

Since $\lambda_{i}-\mu_{j}$ is not a root of $\mathfrak{s o}(10)$, we have $\left[V_{\lambda_{1}} \oplus V_{\lambda_{2}}, V_{\mu_{1}} \oplus V_{\mu_{2}}\right]=0$. Moreover, $\lambda_{1}-\lambda_{2}=\theta_{1}+\theta_{2}$, and $\mu_{1}-\mu_{2}=\theta_{1}-\theta_{2}$, so that the Lie algebras $\mathfrak{g}_{1}^{\prime}$ and $\mathfrak{g}_{2}^{\prime}$ generated by $V_{\lambda_{1}} \oplus V_{\lambda_{2}}$ and $V_{\mu_{1}} \oplus V_{\mu_{2}}$, respectively, satisfy $\mathfrak{g}_{1}^{\prime} \cap(\mathfrak{s o}(10) \oplus \mathfrak{u}(1))=\left\langle\mathfrak{g}_{ \pm\left(\theta_{1}+\theta_{2}\right)}\right\rangle \oplus \mathfrak{t}_{1}^{1}$ and $\mathfrak{g}_{2}^{\prime} \cap(\mathfrak{s o}(10) \oplus \mathfrak{u}(1))=\left\langle\mathfrak{g}_{ \pm\left(\theta_{1}-\theta_{2}\right)}\right\rangle \oplus \mathfrak{t}_{2}^{1}$, i.e., both are isomorphic to $\mathfrak{u}(2)$ and act on $V_{\lambda_{1}} \oplus V_{\lambda_{2}}$ and $V_{\mu_{1}} \oplus V_{\mu_{2}}$, respectively, via the standard representation on $\mathbb{C}^{2}$. Thus, we have the following:

i. $\left(\mathfrak{g}_{i}^{\prime}, \mathfrak{g}_{i}^{\prime} \cap(\mathfrak{s o}(10) \oplus \mathfrak{u}(1))\right)$ is a symmetric pair congruent to $(\mathfrak{s u}(3), \mathfrak{u}(2))$,

ii. $\left[\mathfrak{g}_{1}^{\prime}, \mathfrak{g}_{2}^{\prime}\right]=0$, i.e., $\mathfrak{g}^{\prime}:=\mathfrak{g}_{1}^{\prime} \oplus \mathfrak{g}_{2}^{\prime} \cong \mathfrak{s u}(3) \oplus \mathfrak{s u}(3)$

iii. $\mathfrak{g}^{\prime} \cap(\mathfrak{s o}(10) \oplus \mathfrak{u}(1))=\mathfrak{s o}(4) \oplus \mathfrak{u}(1)$ so that $\mathfrak{k}_{0} \cap \mathfrak{g}^{\prime} \cong \mathfrak{s o}(4)$, which is included into $\mathfrak{s o}(10)$ by the standard embedding, and into $\mathfrak{g}^{\prime}$ as $\mathfrak{k}_{0} \cap \mathfrak{g}^{\prime} \cong \mathfrak{s u}(2) \oplus \mathfrak{s u}(2) \hookrightarrow$ $\mathfrak{s u}(3) \oplus \mathfrak{s u}(3)$.

Furthermore, since $\mathfrak{h}_{0} \subset \mathfrak{s o}(10)$ is the Lie algebra which stabilizes $\theta_{i}$, it follows that $\mathfrak{h}_{0} \cap \mathfrak{g}^{\prime} \subset \mathfrak{k}_{0} \cap \mathfrak{g}^{\prime}$ is the standard inclusion $\mathfrak{s o}(3) \subset \mathfrak{s o}(4)$ which corresponds to $\triangle \mathfrak{s u}(2) \subset \mathfrak{s u}(2) \oplus \mathfrak{s u}(2)$ as asserted.

(b) Suppose $(r, k)=(7,6)$, corresponding to the quaternionic symmetric space $E_{7} / \operatorname{Spin}(12) \cdot \operatorname{Sp}(1)$. Then $(\mathfrak{s o}(12) \oplus \mathfrak{s p}(1))^{\perp}$ is a quaternionic representation of $\mathfrak{s o}(12) \oplus \mathfrak{s p}(1)$, and since $-\lambda$ is a weight whenever $\lambda$ is, the weight space $W_{\lambda}:=\left(V_{\lambda} \oplus V_{-\lambda}\right) \cap \mathfrak{g} \subset(\mathfrak{s o}(12) \oplus \mathfrak{s p}(1))^{\perp}$ for $\lambda=\frac{1}{2}\left(\varepsilon_{1} \theta_{1}+\ldots+\varepsilon_{r} \theta_{6}\right)$ is well defined as a one-dimensional quaternionic vector space, and $\left[W_{\lambda}, W_{\lambda^{\prime}}\right] \neq 0$ if and only if $\lambda \pm \lambda^{\prime}$ is a root of $\mathfrak{s o}(12)$; moreover, in this case, $\left[W_{\lambda}, W_{\lambda^{\prime}}\right]=\mathfrak{g}_{\lambda \pm \lambda^{\prime}} \oplus \mathfrak{t}^{1} \oplus \mathfrak{s p}(1)$ with $\mathfrak{t}^{1}=\operatorname{span}\left(\lambda, \lambda^{\prime}\right) \cap\left(\lambda \pm \lambda^{\prime}\right)^{\perp}$. We define the Lie algebra $\mathfrak{g}_{1}^{\prime} \subset \mathfrak{e}_{7}$ generated by $W:=W_{\lambda_{1}} \oplus \ldots \oplus W_{\lambda_{4}}$, where

$$
\lambda_{i}:=\frac{1}{2}\left(\varepsilon_{1} \theta_{1}+\varepsilon_{2} \theta_{2}+\varepsilon_{3} \theta_{3}+\theta_{4}+\theta_{5}+\theta_{6}\right), \text { where } \varepsilon_{k}= \pm 1 \text { and } \varepsilon_{1} \varepsilon_{2} \varepsilon_{3}=1 .
$$

Since $\lambda_{i}+\lambda_{j}$ is never a root of $\mathfrak{s o}(12)$, we have $\mathfrak{g}_{1}^{\prime} \cap(\mathfrak{s o}(12) \oplus \mathfrak{s p}(1))=[W, W]=$ $\left\langle\mathfrak{g}_{ \pm \theta_{i} \pm \theta_{j}}, 1 \leq i<j \leq 3\right\rangle \oplus \mathfrak{t}^{1} \oplus \mathfrak{s p}(1) \cong \mathfrak{s o}(6) \oplus \mathfrak{t}^{1} \oplus \mathfrak{s p}(1) \cong \mathfrak{s u}(4) \oplus \mathfrak{u}(2)$ which acts on $W$ via the tensor representation $\mathbb{C}^{4} \otimes \mathbb{C}^{2}$. Since $\left(\mathfrak{g}_{1}^{\prime}, \mathfrak{g}_{1}^{\prime} \cap(\mathfrak{s o}(12) \oplus \mathfrak{s p}(1))\right)$ is a symmetric pair, it follows that $\mathfrak{g}_{1}^{\prime} \cong \mathfrak{s u}(6)$ with the inclusion $\mathfrak{g}_{1}^{\prime} \cap(\mathfrak{s o}(12) \oplus \mathfrak{s p}(1))=$ $\mathfrak{s}(\mathfrak{u}(4) \oplus \mathfrak{u}(2)) \subset \mathfrak{s u}(6)$ and hence, $\mathfrak{k}_{0} \cap \mathfrak{g}_{1}^{\prime}=\mathfrak{u}(4) \subset \mathfrak{s u}(6)$. 
Now $\mathfrak{h}_{0} \cap \mathfrak{g}_{1}^{\prime} \subset \mathfrak{u}(4)$ is the stabilizer of an element of the central extension of the representation of $\mathfrak{s u}(4) \cong \mathfrak{s o}(6)$ on $\mathbb{R}^{6}$. Thus, $\mathfrak{h}_{0} \cap \mathfrak{g}_{1}^{\prime} \cong \mathfrak{t}^{1} \oplus \mathfrak{s o}(5) \cong \mathfrak{t}^{1} \oplus \mathfrak{s p}(2)$ which is embedded in the canonical way as $\mathfrak{t}^{1} \oplus \mathfrak{s p}(2) \subset \mathfrak{t}^{1} \oplus \mathfrak{s u}(4)$.

Thus, we have a subgroup $\mathfrak{g}_{1}^{\prime} \cong \mathfrak{s u}(6)$ with $\mathfrak{k}_{0} \cap \mathfrak{g}_{1}^{\prime}=\mathfrak{u}(4)$ and $\mathfrak{h}_{0} \cap \mathfrak{g}_{1}^{\prime} \cong \mathfrak{t}^{1} \oplus \mathfrak{s p}(2) \subset$ $\mathfrak{u}(4)$, so that the existence of $\mathfrak{g}^{\prime} \cong \mathfrak{s u}(3) \oplus \mathfrak{s u}(3) \subset \mathfrak{g}_{1}^{\prime}$ with the asserted properties follows as in case 3 .

(c) Suppose $(r, k)=(8,8)$, corresponding to the real symmetric space $E_{8} / \operatorname{Spin}(16)$. Then $(\mathfrak{s o}(16))^{\perp}$ is a real representation of $\mathfrak{s o}(16)$, and since $-\lambda$ is a weight whenever $\lambda$ is, the weight space $W_{\lambda}:=\left(V_{\lambda} \oplus V_{-\lambda}\right) \cap \mathfrak{g} \subset(\mathfrak{s o}(16))^{\perp}$ for $\lambda=$ $\frac{1}{2}\left(\varepsilon_{1} \theta_{1}+\ldots+\varepsilon_{r} \theta_{k}\right)$ is well defined as a two-dimensional real vector space, and $\left[W_{\lambda}, W_{\lambda^{\prime}}\right] \neq 0$ if and only if $\lambda \pm \lambda^{\prime}$ is a root of $\mathfrak{s o}(16)$; moreover, in this case, $\left[W_{\lambda}, W_{\lambda^{\prime}}\right]=\mathfrak{g}_{ \pm \lambda \pm \lambda^{\prime}} \oplus \mathfrak{t}^{1}$ where $\mathfrak{t}^{1} \subset \operatorname{span}\left(\lambda, \lambda^{\prime}\right)$. We define the weights

$\lambda_{1 / 2}:=\frac{1}{2}\left( \pm\left(\theta_{1}+\theta_{2}\right)+\theta_{3}+\ldots+\theta_{8}\right), \mu_{1 / 2}:=\frac{1}{2}\left( \pm\left(\theta_{1}-\theta_{2}\right)+\theta_{3}+\theta_{4}+\theta_{5}-\theta_{6}-\theta_{7}-\theta_{8}\right)$.

Since $\lambda_{i} \pm \mu_{j}$ is not a root of $\mathfrak{s o}(16)$, we have, $\left[W_{\lambda_{1}} \oplus W_{\lambda_{2}}, W_{\mu_{1}} \oplus W_{\mu_{2}}\right]=0$, and the Lie algebras $\mathfrak{g}_{1}^{\prime}$ and $\mathfrak{g}_{2}^{\prime}$ generated by $W_{\lambda_{1}} \oplus W_{\lambda_{2}}$ and $W_{\mu_{1}} \oplus W_{\mu_{2}}$, respectively, satisfy $\mathfrak{g}_{1}^{\prime} \cap \mathfrak{s o}(16)=\mathfrak{g}_{ \pm\left(\theta_{1}+\theta_{2}\right)} \oplus \mathfrak{t}_{1}^{1} \cong \mathfrak{u}(2)$ and $\mathfrak{g}_{2}^{\prime} \cap \mathfrak{s o}(16)=\mathfrak{g}_{ \pm\left(\theta_{1}-\theta_{2}\right)} \oplus \mathfrak{t}_{2}^{1} \cong \mathfrak{u}(2)$, which act on $W_{\lambda_{1}} \oplus W_{\lambda_{2}}$ and $W_{\mu_{1}} \oplus W_{\mu_{2}}$, respectively, via the standard representation.

Just as in case (a), it now follows that $\mathfrak{g}_{i}^{\prime} \cong \mathfrak{s u}(3)$ with $\left[\mathfrak{g}_{1}^{\prime}, \mathfrak{g}_{2}^{\prime}\right]=0$, so that for $\mathfrak{g}^{\prime}:=\mathfrak{g}_{1}^{\prime} \oplus \mathfrak{g}_{2}^{\prime}$ we have $\mathfrak{k}_{0} \cap \mathfrak{g}^{\prime}=\mathfrak{u}(2) \oplus \mathfrak{u}(2)$, and $\mathfrak{h}_{0} \cap \mathfrak{g}^{\prime}=\triangle \mathfrak{s u}(2) \oplus \mathfrak{z}(\mathfrak{u}(2) \oplus \mathfrak{u}(2)) \subset$ $\mathfrak{u}(2) \oplus \mathfrak{u}(2)$ as asserted.

Proposition 5.8 Let $H \subset K \subset G$ be a triple corresponding to the second entry of Table 3 such that (4) is satisfied.

Then there are compact Lie groups $H^{\prime} \subset G^{\prime}$ such that $G=G_{2} \times G^{\prime}, K=S O(4) \times H^{\prime}$ and $H=\left(S U(2)_{1} \cdot T\right) \times H^{\prime}$, where $T \subset S U(2)_{3}$ is at most one dimensional.

Proof. Since in this case $\mathfrak{g}_{2} \triangleleft \mathfrak{g}$, it follows that $G=\left(G_{2} \times G^{\prime}\right) / \Gamma$ for some finite subgroup $\Gamma \subset Z\left(G_{2} \times G^{\prime}\right)=Z\left(G_{2}\right) \times Z\left(G^{\prime}\right)$. But $Z\left(G_{2}\right)=1$ so that $\Gamma \subset G^{\prime}$, and replacing $G^{\prime}$ by $G^{\prime} / \Gamma$ we have $G=G_{2} \times G^{\prime}$.

Now $K_{0}=S U(2)_{3} \subset K$, and $H=T \cdot \tilde{H}$ where $T \subset K_{0}$ is at most one dimensional and $\tilde{H} \subset Z_{G} S U(2)_{3}=S U(2)_{1} \times G^{\prime}$ is the ineffective kernel of the action of $K$ on $S^{2}$ or $S^{3}$, respectively. Thus, the proposition follows if we can show that $S U(2)_{1} \subset \tilde{H} \subset H$ or, equivalently, $\mathfrak{s u}(2)_{1} \subset \mathfrak{h}$.

As in STa, we decompose the Lie algebra $\mathfrak{g}_{2}$ according to the symmetric pair decomposition of $G_{2} / S O(4)$ as

$$
\mathfrak{g}_{2}=\left(\mathfrak{s p}(1)_{3} \oplus \mathfrak{s p}(1)_{1}\right) \oplus \mathbb{H}^{2},
$$

where $\mathfrak{s p}(1)_{3} \subset \mathfrak{s p}(2)$ is the Lie algebra spanned by

$$
E_{0}:=\left(\begin{array}{cc}
3 i & \\
& i
\end{array}\right), E_{+}:=\left(\begin{array}{cc}
0 & \sqrt{3} \\
-\sqrt{3} & 2 j
\end{array}\right), E_{-}:=\left(\begin{array}{cc}
0 & \sqrt{3} i \\
\sqrt{3} i & 2 k
\end{array}\right)
$$


and acts on $\mathbb{H}^{2}$ from the left, whereas $\mathfrak{s p}(1)_{1}=\operatorname{Im}(\mathbb{H})$ acts via scalar multiplication from the right. Indeed, one verifies the bracket relations

$$
\left[E_{0}, E_{ \pm}\right]= \pm 2 E_{\mp} \text {, and }\left[E_{+}, E_{-}\right]=2 E_{0} .
$$

Evidently, $\mathbb{H}^{2} \subset \mathfrak{s}$. Moreover, since $\mathfrak{t} \subset \mathfrak{s u}(2)_{3}$ is at most one dimensional, we may conjugate $K$ by an appropriate element of $S U(2)_{3}$ and assume w.l.o.g. that $\mathfrak{t} \subset \mathbb{R} E_{+}$so that $E_{0}, E_{-} \in \mathfrak{m}$.

Suppose that $\mathfrak{s u}(2)_{1} \subsetneq \mathfrak{h}$. Then there must be an element $s=s_{1}+s^{\prime} \in \mathfrak{s}$ with $s^{\prime} \in \mathfrak{g}^{\prime}$ and $0 \neq s_{1} \in \mathfrak{s u}(2)_{1}$. Again, after conjugating $K$ by an appropriate element of $S U(2)_{1}$ and rescaling $s$, we may assume w.l.o.g. that $a d_{s}: \mathbb{H}^{2} \rightarrow \mathbb{H}^{2}$ corresponds to right multiplication by $i \in \operatorname{Im}(\mathbb{H})$. We let

$$
e_{1}:=\left(\begin{array}{l}
1 \\
0
\end{array}\right) \text { and } e_{2}:=\left(\begin{array}{l}
0 \\
1
\end{array}\right) \in \mathbb{H}^{2}
$$

be the standard basis. We assert that $\left[e_{1}, e_{2}\right]=\lambda E_{+}$for some $0 \neq \lambda \in \mathbb{R}$. To see this, note that $\left[e_{1}, e_{2}\right] \in \mathfrak{s u}(2)_{3} \oplus \mathfrak{s u}(2)_{1}$ since $\left(\mathfrak{g}_{2}, \mathfrak{s u}(2)_{3} \oplus \mathfrak{s u}(2)_{1}\right)$ is a symmetric pair. Moreover, for $q \in \mathfrak{s u}(2)_{1} \cong \operatorname{Im}(\mathbb{H})$ we have

$$
\begin{aligned}
Q\left(q,\left[e_{1}, e_{2}\right]\right)=Q\left(\left[q, e_{1}\right], e_{2}\right) & =Q\left(\left(\begin{array}{c}
q \\
0
\end{array}\right),\left(\begin{array}{l}
0 \\
1
\end{array}\right)\right)=0, \\
Q\left(E_{0},\left[e_{1}, e_{2}\right]\right)=Q\left(\left[E_{0}, e_{1}\right], e_{2}\right) & =Q\left(\left(\begin{array}{c}
3 i \\
0
\end{array}\right),\left(\begin{array}{c}
0 \\
1
\end{array}\right)\right)=0, \\
Q\left(E_{-},\left[e_{1}, e_{2}\right]\right)=Q\left(\left[E_{-}, e_{1}\right], e_{2}\right) & =Q\left(\left(\begin{array}{c}
0 \\
\sqrt{3} i
\end{array}\right),\left(\begin{array}{c}
0 \\
1
\end{array}\right)\right)=0, \\
Q\left(E_{+},\left[e_{1}, e_{2}\right]\right)=Q\left(\left[E_{+}, e_{1}\right], e_{2}\right) & =Q\left(\left(\begin{array}{c}
0 \\
-\sqrt{3}
\end{array}\right),\left(\begin{array}{c}
0 \\
1
\end{array}\right)\right)=-\sqrt{3}\left|e_{2}\right|_{Q}^{2} \neq 0,
\end{aligned}
$$

since $\left.Q\right|_{\mathbb{H}^{2}}$ must be a multiple of the standard inner product by irreducibility of $G_{2} / S O(4)$. Now let us define the following sequence $X_{n}, Y_{n} \in \mathfrak{m} \oplus \mathfrak{s}$.

$$
X_{n}:=E_{0}-3 s-\frac{2}{\lambda n} e_{2}, \text { and } Y_{n}:=E_{-}+n e_{1}
$$

for $0 \neq \lambda \in \mathbb{R}$ from above. Note that

$$
\left[E_{0}-3 s, e_{1}\right]=E_{0} e_{1}-3 e_{1} i=0,
$$

hence

$$
\begin{aligned}
{\left[X_{n}, Y_{n}\right] } & =\left[E_{0}-3 s-\frac{2}{\lambda n} e_{2}, E_{-}+n e_{1}\right]=-2 E_{+}+\frac{2}{\lambda n} E_{-} e_{2}-\frac{2}{\lambda} \underbrace{\left[e_{2}, e_{1}\right]}_{=-\lambda E_{+}} \\
& =\frac{2}{\lambda n} E_{-} e_{2} .
\end{aligned}
$$

Thus, $\lim \left[X_{n}, Y_{n}\right]=0$ whereas $\left(X_{n}\right)_{\mathfrak{m}} \wedge\left(Y_{n}\right)_{\mathfrak{m}}=E_{0} \wedge E_{-} \neq 0$ is constant. This violates (4) and gives the desired contradiction. 
Remark 5.9 If we consider $G:=G_{2} \times S U(2)$ and $H=\triangle S U(2) \subset S U(2)_{1} \times S U(2) \subset$ $G_{2} \times S U(2)$, and $K=H \times S U(2)_{3}$, then (4) is violated by Proposition 5.8. On the other hand, one can show that there are no $X, Y \in \mathfrak{m} \oplus \mathfrak{s}$ with $X_{\mathfrak{m}} \wedge Y_{\mathfrak{m}} \neq 0$ and $[X, Y]=0$. That is, condition (4) cannot be weakened to the property that $[X, Y]=0$ only if $X_{\mathfrak{m}}, Y_{\mathfrak{m}}$ are linearly dependent.

We call a homogeneous vector bundle $M=G \times_{K} V$ G-reducible if there is a non-trivial decomposition of the Lie algebra $\mathfrak{g}=\mathfrak{g}_{1} \oplus \mathfrak{g}_{2}$ such that $\mathfrak{k}=\mathfrak{k}_{1} \oplus \mathfrak{k}_{2}$ where $\mathfrak{k}_{i}:=\mathfrak{k} \cap \mathfrak{g}_{i}$ and such that $\mathfrak{k}_{2} \subsetneq \mathfrak{g}_{2}$ acts trivially on $V$. Otherwise, we call $M G$-irreducible.

If $M$ is reducible, then - allowing for an ineffective action - we may assume that $G=$ $G_{1} \times G_{2}$. Moreover, after replacing $M$ by a finite $G$-equivariant cover $\tilde{M}$, we may assume that $K$ is connected and hence $K=K_{1} \times K_{2}$ with $K_{i}:=K \cap G_{i}$, and $K_{2} \subset K$ acts trivially on $V$. Thus, $\tilde{M}=\left(G_{2} / K_{2}\right) \times M^{\prime}$ where $M^{\prime}=G_{1} \times_{K_{1}} V$ and $\operatorname{dim}\left(G_{2} / K_{2}\right)>0$, and $\tilde{M}$ has a $G$-invariant metric of nonnegative curvature with normal homogeneous collar if and only if $M^{\prime}$ does. Thus, it is natural to assume that $M$ is $G$-irreducible.

Proof of Theorems 1.1- 1.5 It was already shown in section 2 that the bundles asserted in these theorems admit invariant nonnegatively curved metrics with normal homogeneous collar, so it remains to show that there cannot be any others. In particular, we may assume from now on that the rank of the disk bundle is $\geq 3$ and $\neq 4$ so that, in particular, $\mathfrak{m}_{1} \neq 0$.

If $L \subset G$ is a normal subgroup, then after replacing $G$ by a finite cover, we may assume that $G=G^{\prime} \times L$. Since $L \subset K$, we must have $K=H^{\prime} \times L$ for some subgroup $H^{\prime} \subset G^{\prime}$. Moreover, $L \subset O(V)$ acts transitively on the unit sphere, so that $M=\left(G^{\prime} \times L\right) \times_{H^{\prime} \times L} V$ is essentially trivial.

If $L \subset G$ is not normal and $L \subsetneq \tilde{L}$, then, since we assume that rank to be $\neq 4$ and thus, $L \neq S U(2) \cdot S U(2)$, Proposition [5.6 implies that $\tilde{L} \subset G$ is normal, whence $G=\tilde{L} \cdot G^{\prime}$. Replacing $G$ and hence $\tilde{L}$ by a finite cover, we may assume that $\tilde{L}$ is simply connected. Thus, by Proposition 5.5. we have $L=\operatorname{Spin}(n) \subset \operatorname{Spin}(m)=\tilde{L}$ for $(n, m) \in\{(6,7),(7,8),(7,9)\}$, where in either case $L$ acts on $S^{7}$ so that $M \rightarrow G / K$ is a bundle of rank 8 .

We have $K=K_{0} \cdot H^{\prime}=\operatorname{Spin}(n) \cdot T \cdot H^{\prime}$ where $\operatorname{dim} T \leq 1$, and $T=S^{1}$ is possible only for $(n, m)=(6,7)$. Thus, $T \cdot H^{\prime} \subset Z_{\operatorname{Spin}(m)}(\operatorname{Spin}(n)) \cdot G^{\prime}$ where $Z$ denotes the centralizer.

If $(n, m)=(6,7)$ or $(7,8)$, then $\left(Z_{\operatorname{Spin}(m)} \operatorname{Spin}(n)\right)_{0}=1$, hence $T \cdot H^{\prime} \subset G^{\prime}$. If $T=1$ then the condition of $G$-irreducibility implies that $G^{\prime}=1$, which is the second case for $p=0$ and the third case of Theorem 1.2, respectively. If $T=S^{1}$ then $(n, m)=(6,7)$ which corresponds to case $4(\mathrm{c})$ of that theorem.

If $(n, m)=(7,9)$, then $T=1$ and $H^{\prime} \subset Z_{\operatorname{Spin}(9)} \operatorname{Spin}_{0}(7) \cdot G^{\prime}=\operatorname{Spin}(2) \cdot G^{\prime}$. The case $H^{\prime}=1$ is the second case of Theorem 1.2 with $p=1$. If $H^{\prime} \neq 1$, then by $G$-irreducibility, we have $H^{\prime} \not \subset G^{\prime}$, corresponding to case 4(b) of Theorem 1.2.

Finally, suppose that $L=\tilde{L} \subset G$ is not normal and different from $S U(2) \cdot S U(2)$. Then by Proposition 5.7 there must be a normal subgroup $G_{1} \subset G$ containing $L$ which is either $S U(5)$, $G_{2}, S p(p+1)$ for $p \geq 1$, or $\operatorname{Spin}(p+9)$ for $p \in\{0,1,2\}$. In either case, $L=S p(1) \cong S U(2)$ acting on $S^{2}$ or $S^{3}, L=S U(4) \cong \operatorname{Spin}(6)$ acting on $S^{5}$ or $L=\operatorname{Spin}(8)$ acting on $S^{7}$ by the 
spin representation which shows that the rank is as asserted in Theorem 1.1. Furthermore, $K_{0}=L$ and hence, $K=L \cdot H^{\prime}$ with $H^{\prime} \subset N_{\text {orm }} L$.

If $G_{1}=S U(5)$ so that $G=S U(5) \cdot G^{\prime}$, then $H^{\prime} \subset N_{\text {orm }} L=S^{1} \cdot G^{\prime}$. If $H^{\prime}=1$ then this is the first case of Theorem 1.3, otherwise, we get the second case of that theorem.

If $G_{1}=G_{2}$, then by Proposition [5.8, we must have $S U(2)_{1} \subset H$, and from there, the hypothesis of $G$-irreducibility implies that $G=G_{2}$, which yields the first entry in Theorem 1.4.

If $G_{1}=S p(p+1)$ so that $G=S p(p+1) \cdot G^{\prime}$, then $H^{\prime} \subset \operatorname{Norm}_{G} L=S p(p) \cdot G^{\prime}$ which corresponds to the second entry in Theorem 1.4.

Finally, in the last case, $G_{1}=\operatorname{Spin}(p+9)$ so that $G=\operatorname{Spin}(p+9) \cdot G^{\prime}$ and $K=\operatorname{Spin}(8) \cdot H^{\prime}$ with $H^{\prime} \subset Z_{G} \operatorname{Spin}(8)=\operatorname{Spin}(p+1) \cdot G^{\prime}$. If $H^{\prime}=1$ then this corresponds to the first case of Theorem 1.2, the general case is listed in $4(\mathrm{a})$ of that theorem.

\section{References}

[Ba] J. Baez, The octonions, Bull. Amer. Math. Soc. (N.S.) 39, No. 2 (2002) 145-205; Errata for: "The octonions", Bull. Amer. Math. Soc. (N.S.) 42 (2005), no. 2, 213 (electronic)

[Be] M. Berger, Les variétés riemanniennes homogènes normales simplement connexes à courbure strictement positive, Ann. Scuola Norm. Sup. Pisa 15 (1961) 179-246

[C] J. Cheeger, Some examples of manifolds of nonnegative curvature, J. Diff. Geom. 8 (1973) $623-628$

[GVWZ] K. Grove, L. Verdiani, B. Wilking, W. Ziller, Nonnegative curvature obstructions in cohomogeneity one and the Kervaire spheres, Ann. Sc. Norm. Super. Pisa, Cl. Sci. (5) 5, No. 2 (2006) 159-170

[GWZ] K. Grove, B. Wilking, W. Ziller, Positively curved cohomogeneity one manifolds and 3Sasakian geometry, J. Diff. Geom. 78 (2008) 33-111.

[GZ] K. Grove and W. Ziller, Curvature and symmetry of Milnor spheres, Ann. of Math. (2) 152 (2000) 331-36

[H] S. Helgason, Differential Geometry, Lie Groups, and Symmetric Spaces, Graduate Studies in Math., Springer Verlag

[MS] D. Montgomery and H. Samelson, Transformation groups of spheres, Ann. Math 44 (1943) $454-470$

[P] G. Perelman, Proof of the soul conjecture of Cheeger and Gromoll, J. Differential Geom. 40 (1994), 209-212.

[STa] L. Schwachhöfer and K. Tapp, Homogeneous Metrics with nonnegative curvature, preprint, arXiv:0804.3729

[STu] L. Schwachhöfer and W. Tuschmann, Almost nonnegative curvature and cohomogeneity one, Preprint no. 62/2001, Max-Planck-Institut für Mathematik in den Naturwissenschaften Leipzig, http://www.mis.mpg.de/cgi-bin/preprints.pl 
[Z] W.Ziller, Examples of Riemannian manifolds with nonnegative sectional curvature, Metric and Comparison Geometry, Surv. Diff. Geom. 11, ed. K.Grove and J.Cheeger, Intern. Press, 2007

Fakultät Für Mathematik, Technische Universität Dortmund, Vogelpothsweg 87, 44227 Dortmund, Germany

E-mail address: lschwach@math.uni-dortmund.de

Department of Mathematics, Saint Joseph University, 5600 City Avenue PhiladelPHIA, PA 19131, USA

E-mail address: ktapp@sju.edu 